\title{
Spectral action for Bianchi type-IX cosmological models
}

\author{
Wentao Fan, Farzad Fathizadeh and Matilde Marcolli \\ Division of Physics, Mathematics and Astronomy, California Institute of Technology, \\ 1200 E. California Blvd., Pasadena, CA 91125, U.S.A. \\ E-mail: wffan@caltech.edu, farzadf@caltech.edu, matilde@caltech.edu
}

\begin{abstract}
A rationality result previously proved for Robertson-Walker metrics is extended to a homogeneous anisotropic cosmological model, namely the Bianchi type-IX minisuperspace. It is shown that the Seeley-de Witt coefficients appearing in the expansion of the spectral action for the Bianchi type-IX geometry are expressed in terms of polynomials with rational coefficients in the cosmic evolution factors $w_{1}(t), w_{2}(t), w_{3}(t)$, and their higher derivates with respect to time. We begin with the computation of the Dirac operator of this geometry and calculate the coefficients $a_{0}, a_{2}, a_{4}$ of the spectral action by using heat kernel methods and parametric pseudodifferential calculus. An efficient method is devised for computing the Seeley-de Witt coefficients of a geometry by making use of Wodzicki's noncommutative residue, and it is confirmed that the method checks out for the cosmological model studied in this article. The advantages of the new method are discussed, which combined with symmetries of the Bianchi type-IX metric, yield an elegant proof of the rationality result.
\end{abstract}

KeYwords: Non-Commutative Geometry, Models of Quantum Gravity

ARXiv EPrint: 1506.06779 


\section{Contents}

1 Introduction 1

2 The Dirac operator of Bianchi type-IX metrics 5

3 Calculation of the terms up to $a_{4}$ in the spectral action $\quad 8$

4 Heat coefficients and the Wodzicki residue 12

5 Rationality of the spectral action for Bianchi type-IX metrics $\quad 18$

6 Gravitational instantons, modular forms, and rationality 20

$\begin{array}{lll}7 & \text { Conclusions } & 22\end{array}$

A Pseudodifferential symbol of $D^{2} \quad 22$

B Full expression of the term $a_{4}\left(D^{2}\right) \quad 24$

\section{Introduction}

Quantum cosmology studies the early universe where the energy scale is so high that one would need to incorporate into the theory both quantum gravity and the Standard Model with unbroken symmetries such as the hypothetical supersymmetry. This makes the exact solution of any quantum cosmological problem essentially impossible as it amounts to solving quantum fields genuinely interacting with quantized gravity, the nature of which we know little about. However, since we are only interested in the large-scale behavior of the early universe whose mass-energy distribution is highly homogeneous, we are encouraged to exploit this symmetry and focus only on a few long-wavelength degrees of freedom. In quantum cosmology, this common practice is known as the minisuperspace approximation, which can be rigorously justified under certain criteria $[37,51]$.

Well-known examples of minisuperspaces include the Robertson-Walker model and its anisotropic generalization to the Bianchi type-IX model. The Robertson-Walker metric is of the form

$$
d s^{2}=d t^{2}+a(t)^{2} d \sigma^{2}
$$

where $a(t)$ is a general cosmic factor of the expanding universe and $d \sigma^{2}$ is the round metric on the 3 -sphere $\mathbb{S}^{3}$. The Bianchi type-IX model, which enjoys a reduced $\mathrm{SU}(2)$ isometry group rather than the full $\mathbb{S}^{3}$ symmetry, is written as

$$
d s^{2}=w_{1}(t) w_{2}(t) w_{3}(t) d t^{2}+\frac{w_{2}(t) w_{3}(t)}{w_{1}(t)} \sigma_{1}^{2}+\frac{w_{3}(t) w_{1}(t)}{w_{2}(t)} \sigma_{2}^{2}+\frac{w_{1}(t) w_{2}(t)}{w_{3}(t)} \sigma_{3}^{2},
$$

where $\sigma_{i}$ are left-invariant 1-forms on $\mathrm{SU}(2)$-orbits. 
In quantum cosmology, according to the Hartle-Hawking proposal (see [19, 24]), a path integral approach is formulated in terms of a sum over 4-dimensional geometries, with an action functional determined by the Einstein-Hilbert action (or a variant thereof) for Euclidean gravity on the 4-dimensional geometries. The contribution of most geometries is negligible and, in a semi-classical approximation, the path integral concentrates on the instanton geometries. In particular, the minisuperspace models, which are based on spatially homogeneous 3-dimensional geometries, have the advantage that the metric structure is encoded in a finite dimensional problem, and the instanton equations reduce in the Bianchi IX case to a well known system of singular ordinary differential equation, the Painlevé VI equations, see [32], and also [3, 49, 53].

This paper is the first part of an ongoing investigation on a new approach to quantum cosmology, based on the spectral action functional. In particular, here we focus on explicitly computing the spectral action functional for the Bianchi IX minisuperspace models. In the forthcoming second part [23] we will crucially use the explicit formulae that we obtained in this paper to compute the spectral action for those Bianchi IX models that are also self-dual, namely the gravitational instantons. In particular, the rationality result that we prove in this paper will play a crucial role in obtaining an explicit expression of the spectral action of the gravitational instantons in terms of modular forms. This will lead to a surprising occurrence in quantum cosmology of the vector valued modular forms considered in the Eichler-Zagier theory of Jacobi forms [21].

The spectral action functional, [7, 13], is an action functional for Euclidean gravity, which can be regarded as a modified gravity model. It is defined in terms of the spectrum of the Dirac operator, and it recovers the usual Einstein-Hilbert action with cosmological term, along with modified gravity terms (Weyl curvature conformal gravity, Gauss-Bonnet gravity), in an asymptotic expansion in the energy scale. As a model of gravity, the spectral action was used in recent years as a possible source of new early universe models and inflationary mechanisms, [4, 22, 36, 42-48].

More precisely, in this approach all the information about the gravitational field is encoded in and recovered from the Dirac operator. The spectral action is defined as Trace $(f(D / \Lambda))$, where $D$ is the Dirac operator of the 4-dimensional geometry, $\Lambda$ is an energy scale, and $f$ is a positive even function on the real line, a smooth approximation to a cutoff function. This functional has an asymptotic expansion for large $\Lambda$ of the form $[7,18]$

$$
\operatorname{Trace}(f(D / \Lambda)) \sim \sum_{\beta \in \Pi} f_{\beta} \Lambda^{\beta} f|D|^{-\beta}+f(0) \zeta_{D}(0)+\cdots,
$$

where the sum is over points $\beta \in \mathbb{R}_{+}$that belong to the dimension spectrum (the set of poles of the zeta functions $\zeta_{D}(s)$ of the Dirac operator, and other related zeta functions), and the contribution $f|D|^{-\beta}$ of each of these points is a residue, which is given by an integration of certain explicit curvature expressions. The coefficients $f_{\beta}$ are the momenta of the function $f$ and can be thought of as parameters of the model. In the case of a 4-dimensional compact Riemannian manifold, the contributions of $\beta=0,2,4$ in the expansion recover the EinsteinHilbert action with cosmological term, together with Weyl curvature conformal gravity and a Gauss-Bonnet term. The momenta $f_{\beta}$ give the (effective) gravitational and cosmological 
constants of the model. For a $\operatorname{spin}^{c}$ manifold $M$, the coefficients of this expansion are determined by the Seeley-de Witt coefficients $a_{2 n}\left(D^{2}\right)$ appearing in the heat expansion,

$$
\operatorname{Trace}\left(e^{-t D^{2}}\right) \sim t^{-\operatorname{dim}(M) / 2} \sum_{n=0}^{\infty} a_{2 n}\left(D^{2}\right) t^{n} \quad\left(t \rightarrow 0^{+}\right) .
$$

For a detailed mathematical treatment of the above asymptotic expansion, we refer the reader to chapter 1 of the book [18].

An advantage of the spectral action, with respect to other gravity action functionals, is that it continues to make sense when the underlying geometry becomes a noncommutative geometry, [14]. Indeed, a good analog of compact Riemannian manifolds in the noncommutative world is given by the theory of spectral triples. These are specified by data $(\mathcal{A}, \mathcal{H}, D)$, where $\mathcal{A}$ is an involutive algebra represented by bounded operators on a Hilbert space $\mathcal{H}$, and $D$ is an unbounded self-adjoint operator in $\mathcal{H}$ that plays the role of the Dirac operator, by encoding the metric information. This set up, which includes a great variety of noncommutative spaces, generalizes Riemannian geometry, since Connes' reconstruction theorem [16] states that if $\mathcal{A}$ is commutative, then, under suitable regularity conditions, the triple consists of the algebra of smooth function on a $\operatorname{spin}^{c}$ manifold acting on the $L^{2}$-spinors and $D$ is the Dirac operator. Extending the gravity action functional to noncommutative spaces is especially useful as a method for constructing models of gravity coupled to matter. These are obtained by taking a product of the ordinary 4-dimensional spacetime geometry with a finite noncommutative geometry that specifies the matter content of the model. Then gravity (modelled by the spectral action) on the product "almost-commutative geometry" gives, in the asymptotic expansion, a model of gravity non-minimally coupled to matter on the curved background given by the 4-geometry, [6, 13]. Indeed, in addition to extracting local geometric information and recovering the Einstein-Hilbert action, the spectral action, generalized to suitable almost-commutative geometries, recovers (extensions of) the Standard Model of elementary particles, including gauge fields with the Yang-Mills actions, a Higgs sector, and a fermion content that includes right handed neutrinos with Majorana masses, and a candidate inflation field [6, 8-10, 12, 15, 43, 45].

In this paper we focus only on pure gravity, hence we consider the spectral action on an ordinary, commutative geometry rather than its extension to almost-commutative geometries. However, the model that we discuss here can be further enriched by coupling to a matter sector, by considering different possible almost-commutative geometries over the Bianchi-IX spacetimes.

Beside trying to fit the Standard model into the early universe spacetime, another possible investigation of physical importance is to quantize supersymmetric systems in a Bianchi-type IX minisuperspace. Since the energy scale of the early universe is very likely to be higher than the supersymmetry breaking scale, it is of great interest to see how supersymmetry may possibly change the picture of the early universe. Since the Hamiltonians of supersymmetric systems can often be identified with well-behaving elliptic operators acting on spin bundles for Majorana fermions or fields of differential forms for Dirac fermions, the spectral action [7] approach appears to be a handy tool, where the celebrated Atiyah-Singer 
index theorem may be used to calculate the Witten indices of certain supersymmetric theories and thus to conclude whether the supersymmetry can be spontaneously broken $[2,56]$. We also do not explicitly discuss these aspects in the present paper.

Our main focus here is the explicit computation of the terms in the expansion of the spectral action for a Bianchi-type IX minisuperspace. Carrying out explicit computations of the spectral action functional is usually a very challenging problem: it is of great importance, as a general problem, to develop different methods for computing the spectral action. In particular, any interpretation of the full expansion of a spectral action is highly desirable. For example, using the Poisson summation formula, the spectral action for the Dirac operator on highly symmetric manifolds, such as products of spheres by tori, was computed in [10], and generalized to the case of spherical space forms and Bieberbach manifolds in [5, 44, 45, 52]. For the Euclidean Robertson-Walker spacetime, with a general cosmic factor $a(t)$, Chamseddine and Connes have devised an efficient method in [11] for computing the terms of the spectral action, which is based on making the use of the Euler-Maclaurin formula and the Feynman-Kac formula. They computed the terms up to $a_{10}$ in the expansion and made a conjecture, which was addressed in [26] by using pseudodifferential operators and heat kernel techniques. That is, it was shown that a general term in the expansion is described by a polynomial with rational coefficients in $a(t)$ and its derivatives of a certain order.

The Bianchi type-IX minisuperspace case, which is the focus in this paper, is a homogeneous but anisotropic cosmological model. Since the spectral action of a geometry depends on the eigenvalues of the square of its Dirac operator, we explicitly compute the Dirac operator $D$ of the Bianchi type-IX metric in section 2 and derive the pseudodifferential symbol of $D$. Since it has a lengthy expression, the symbol of $D^{2}$ is given the appendix A. In section 3, following a review of the heat kernel method that uses pseudodifferential calculus for the computation of the Seeley-de Witt coefficients [30], we present the calculation of the terms $a_{0}, a_{2}, a_{4}$ in the expansion of the spectral action associated with $D$. The term $a_{4}$ is presented in a short form there, and for the sake of clarity its full expression is given in the appendix B.

We devise a new method for calculating the Seeley de-Witt coefficients of a geometry in terms of noncommutative residues of operators, which extends the result on the realization of the Einstein-Hilbert action as the residue of a power of the Laplacian, see [31, 33, 35]. This method is explained in detail in section 4 and it checks out to give the same result for the calculated terms $a_{0}, a_{2}, a_{4}$ for the Bianchi type-IX metric. Combining the symmetries of the metric with technical properties of pseudodifferential symbols of parametrices of the Laplacians, which significantly simplify in view of the new method using the Wodzicki residue, we prove a rationality result for a general term in the expansion of the spectral action for the Bianchi type-IX metric in section 5. That is, we show that general terms of the expansion are expressed by several variable polynomials with rational coefficients evaluated on $w_{1}(t), w_{2}(t), w_{3}(t)$, and their derivatives of certain orders. In section 6 , we discuss the gravitational instantons, which form an especially interesting class of Bianchi type-IX models, and elaborate on the significance of the rationality for the spectral action in relation to the arithmetic and number theoretic structures in mathematical physics. Our main results and conclusions are summarized in section 7 . 


\section{The Dirac operator of Bianchi type-IX metrics}

The heat kernel method that uses pseudodifferential calculus for computing the Seeleyde Witt coefficients of an elliptic positive operator on a compact manifold relies on the pseudodifferential symbol of the operator in local charts, see chapter 1 of the book [30]. Thus, in this section we compute the Dirac operator $D$ of the Bianchi type-IX metric and thereby obtain its symbol, using which one can calculate the symbol of $D^{2}$.

The most efficient way of computing the Dirac operator of a geometric space is to use an orthonormal coframe $\left\{\theta^{a}\right\}$ for the metric, which, from the definition of $D$, yields

$$
D=\sum_{a} \theta^{a} \nabla_{\theta_{a}}^{S}
$$

where $\nabla^{S}$ is the spin connection of the spin bundle and $\left\{\theta_{a}\right\}$ is the predual of the coframe, cf. page 68 of the book [29]. Since $\nabla^{S}$ is the lift of the Levi-Civita connection $\nabla$ to the spin bundle, one starts with computing the matrix of 1 -forms $\omega=\left(\omega_{b}^{a}\right)$ such that $\nabla=d+\omega$ in terms of $\theta^{a}$ in a local chart $x=\left(x^{\mu}\right) \in U$, which can be lifted to the matrix of the spin connection 1-forms by making use of the Lie algebra isomorphism $\mu: \mathfrak{s o}(m) \rightarrow \mathfrak{s p i n}(m)$ given by

$$
\mu(A)=\frac{1}{4} \sum_{a, b} A^{a b} e_{a} e_{b}, \quad A=\left(A^{a b}\right) \in \mathfrak{s o}(m),
$$

where $m$ is the dimension of the manifold, see Lemma 4.8 on page 59 of [50]. We note that $\left\{e_{a}\right\}$ is the standard basis for $\mathbb{R}^{m}$ considered inside the Clifford algebra of $\mathbb{R}^{m}$ where $\mathfrak{s p i n}(m)$ is spanned linearly by $\left\{e_{a} e_{b} ; a<b\right\}$.

The $\omega_{b}^{a}$ are found uniquely by writing

$$
\nabla \theta^{a}=\sum_{b} \omega_{b}^{a} \otimes \theta^{b}
$$

and by imposing the conditions that characterize the Levi-Civita connection, namely metric-compatibility and torsion-freeness which respectively imply that

$$
\omega_{b}^{a}=-\omega_{a}^{b}, \quad d \theta^{a}=\sum_{b} \omega_{b}^{a} \wedge \theta^{b}
$$

Therefore the Dirac operator is written as

$$
D=\sum_{a, \mu} \gamma^{a} d x^{\mu}\left(\theta_{a}\right) \frac{\partial}{\partial x^{\mu}}+\frac{1}{4} \sum_{a, b, c} \gamma^{c} \omega_{a c}^{b} \gamma^{a} \gamma^{b}
$$

where $\omega_{a c}^{b}$ are defined by

$$
\omega_{a}^{b}=\sum_{c} \omega_{a c}^{b} \theta^{c}
$$

and the matrices $\gamma^{a}$ represent the Clifford action of $\theta^{a}$ on the spin bundle $S$, namely that, they are $k \times k$ matrices, $k=\operatorname{rk}(S)$, which satisfy the relations $\left(\gamma^{a}\right)^{2}=-I$ and $\gamma^{a} \gamma^{b}+\gamma^{b} \gamma^{a}=0$ for $a \neq b$. 
The Dirac operator is a differential operator of order 1, and using the Fourier inversion formula, its action on a spinor $s$ written in the chosen local chart $U$ can be expressed by

$$
\begin{aligned}
D s(x) & =(2 \pi)^{-m / 2} \int e^{i x \cdot \xi} \sigma(D)(x, \xi) \hat{s}(\xi) d \xi \\
& =(2 \pi)^{-m} \iint e^{i(x-y) \cdot \xi} \sigma(D)(x, \xi) s(y) d y d \xi
\end{aligned}
$$

where $\sigma(D): U \times \mathbb{R}^{m} \rightarrow M_{k}(\mathbb{C})$ is the pseudodifferential symbol of the operator, $\hat{s}$ is the component-wise Fourier transform of $s$, and endomorphisms of $S$ are locally identified with $M_{k}(\mathbb{C})$. That is, considering the formula $(2.1)$, the symbol is written as

$$
\sigma(D)(x, \xi)=\sum_{a, \mu} \gamma^{a} d x^{\mu}\left(\theta_{a}\right)\left(i \xi_{\mu+1}\right)+\frac{1}{4} \sum_{a, b, c} \gamma^{c} \omega_{a c}^{b} \gamma^{a} \gamma^{b}
$$

where $x=\left(x^{0}, x^{1}, \ldots, x^{m-1}\right) \in U$ and $\xi=\left(\xi_{1}, \xi_{2}, \ldots, \xi_{m}\right) \in \mathbb{R}^{m}$. In general, the effect of change of coordinates on pseudodifferential symbols suggests that it is natural to consider $\mathbb{R}^{m}$ as the cotangent fibre at point $x$, see for example Lemma 1.3.2 on page 24 of [30].

We go through the above process for the Bianchi type-IX metric,

$$
\begin{aligned}
d s^{2}= & w_{1} w_{2} w_{3} d t d t+\frac{w_{1} w_{2} \cos (\eta)}{w_{3}} d \phi d \psi+\frac{w_{1} w_{2} \cos (\eta)}{w_{3}} d \psi d \phi \\
& +\left(\frac{w_{2} w_{3} \sin ^{2}(\eta) \cos ^{2}(\psi)}{w_{1}}+w_{1}\left(\frac{w_{3} \sin ^{2}(\eta) \sin ^{2}(\psi)}{w_{2}}+\frac{w_{2} \cos ^{2}(\eta)}{w_{3}}\right)\right) d \phi d \phi \\
& +\frac{\left(w_{1}^{2}-w_{2}^{2}\right) w_{3} \sin (\eta) \sin (\psi) \cos (\psi)}{w_{1} w_{2}} d \eta d \phi+\frac{\left(w_{1}^{2}-w_{2}^{2}\right) w_{3} \sin (\eta) \sin (\psi) \cos (\psi)}{w_{1} w_{2}} d \phi d \eta \\
& +\left(\frac{w_{2} w_{3} \sin ^{2}(\psi)}{w_{1}}+\frac{w_{1} w_{3} \cos ^{2}(\psi)}{w_{2}}\right) d \eta d \eta+\frac{w_{1} w_{2}}{w_{3}} d \psi d \psi
\end{aligned}
$$

which is written in the local coordinates $\left(x^{\mu}\right)=(t, \eta, \phi, \psi)$, where $\mathbb{S}^{3}$ is parametrized by the map

$$
(\eta, \phi, \psi) \mapsto\left(\cos (\eta / 2) e^{i(\phi+\psi) / 2}, \sin (\eta / 2) e^{i(\phi-\psi) / 2}\right),
$$

with the parameter ranges $0 \leq \eta \leq \pi, 0 \leq \phi<2 \pi, 0 \leq \psi<4 \pi$. An orthonormal coframe for $d s^{2}$ is given by

$$
\begin{aligned}
& \theta^{0}=\sqrt{w_{1} w_{2} w_{3}} d t \\
& \theta^{1}=\sin (\eta) \cos (\psi) \sqrt{\frac{w_{2} w_{3}}{w_{1}}} d \phi-\sin (\psi) \sqrt{\frac{w_{2} w_{3}}{w_{1}}} d \eta, \\
& \theta^{2}=\sin (\eta) \sin (\psi) \sqrt{\frac{w_{1} w_{3}}{w_{2}}} d \phi+\cos (\psi) \sqrt{\frac{w_{1} w_{3}}{w_{2}}} d \eta, \\
& \theta^{3}=\cos (\eta) \sqrt{\frac{w_{1} w_{2}}{w_{3}}} d \phi+\sqrt{\frac{w_{1} w_{2}}{w_{3}}} d \psi .
\end{aligned}
$$


By explicit calculations in this basis we find that the non-vanishing $\omega_{a c}^{b}$ are determined by the following terms:

$$
\begin{array}{lll}
\omega_{11}^{0}=-\frac{w_{2}\left(w_{1} w_{3}^{\prime}-w_{3} w_{1}^{\prime}\right)+w_{1} w_{3} w_{2}^{\prime}}{2\left(w_{1} w_{2} w_{3}\right)^{3 / 2}}, & \omega_{22}^{0}=-\frac{w_{2}\left(w_{3} w_{1}^{\prime}+w_{1} w_{3}^{\prime}\right)-w_{1} w_{3} w_{2}^{\prime}}{2\left(w_{1} w_{2} w_{3}\right)^{3 / 2}}, \\
\omega_{33}^{0}=-\frac{w_{2}\left(w_{3} w_{1}^{\prime}-w_{1} w_{3}^{\prime}\right)+w_{1} w_{3} w_{2}^{\prime}}{2\left(w_{1} w_{2} w_{3}\right)^{3 / 2}}, & \omega_{23}^{1}=-\frac{w_{1}^{2} w_{2}^{2}-w_{3}^{2}\left(w_{1}^{2}+w_{2}^{2}\right)}{2\left(w_{1} w_{2} w_{3}\right)^{3 / 2}}, \\
\omega_{32}^{1}=-\frac{w_{1}^{2}\left(w_{2}^{2}-w_{3}^{2}\right)+w_{2}^{2} w_{3}^{2}}{2\left(w_{1} w_{2} w_{3}\right)^{3 / 2}}, & \omega_{31}^{2}=-\frac{w_{2}^{2} w_{3}^{2}-w_{1}^{2}\left(w_{2}^{2}+w_{3}^{2}\right)}{2\left(w_{1} w_{2} w_{3}\right)^{3 / 2}} .
\end{array}
$$

Thus we can write the Dirac operator explicitly as

$$
\begin{aligned}
D= & \sum_{a, \mu} \gamma^{a} e_{a}^{\mu} \frac{\partial}{\partial x^{\mu}}+\frac{1}{4 \sqrt{w_{1} w_{2} w_{3}}}\left(\frac{w_{1}^{\prime}}{w_{1}}+\frac{w_{2}^{\prime}}{w_{2}}+\frac{w_{3}^{\prime}}{w_{3}}\right) \gamma^{1} \\
& -\frac{\sqrt{w_{1} w_{2} w_{3}}}{4}\left(\frac{1}{w_{1}^{2}}+\frac{1}{w_{2}^{2}}+\frac{1}{w_{3}^{2}}\right) \gamma^{2} \gamma^{3} \gamma^{4}
\end{aligned}
$$

where the only non-vanishing $e_{a}^{\mu}=d x^{\mu}\left(\theta_{a}\right)$ are:

$$
\begin{aligned}
& e_{0}^{0}=\frac{1}{\sqrt{w_{1} w_{2} w_{3}}}, \\
& e_{1}^{1}=-\frac{\sqrt{w_{1}} \sin (\psi)}{\sqrt{w_{2} w_{3}}}, \quad e_{2}^{1}=\frac{\sqrt{w_{2}} \cos (\psi)}{\sqrt{w_{1} w_{3}}}, \\
& e_{1}^{2}=\frac{\sqrt{w_{1}} \csc (\eta) \cos (\psi)}{\sqrt{w_{2} w_{3}}}, \quad e_{2}^{2}=\frac{\sqrt{w_{2}} \csc (\eta) \sin (\psi)}{\sqrt{w_{1} w_{3}}}, \quad e_{1}^{3}=-\frac{\sqrt{w_{1}} \cot (\eta) \cos (\psi)}{\sqrt{w_{2} w_{3}}}, \\
& e_{2}^{3}=-\frac{\sqrt{w_{2}} \cot (\eta) \sin (\psi)}{\sqrt{w_{1} w_{3}}}, \quad e_{3}^{3}=\frac{\sqrt{w_{3}}}{\sqrt{w_{1} w_{2}}} .
\end{aligned}
$$

We note that we use the following gamma matrices in our calculations:

$$
\gamma^{0}=\left(\begin{array}{cccc}
0 & 0 & i & 0 \\
0 & 0 & 0 & i \\
i & 0 & 0 & 0 \\
0 & i & 0 & 0
\end{array}\right), \quad \gamma^{1}=\left(\begin{array}{cccc}
0 & 0 & 0 & 1 \\
0 & 0 & 1 & 0 \\
0 & -1 & 0 & 0 \\
-1 & 0 & 0 & 0
\end{array}\right), \quad \gamma^{2}=\left(\begin{array}{cccc}
0 & 0 & 0 & -i \\
0 & 0 & i & 0 \\
0 & i & 0 & 0 \\
-i & 0 & 0 & 0
\end{array}\right), \quad \gamma^{3}=\left(\begin{array}{cccc}
0 & 0 & 1 & 0 \\
0 & 0 & 0 & -1 \\
-1 & 0 & 0 & 0 \\
0 & 1 & 0 & 0
\end{array}\right) \text {. }
$$

Accordingly, the pseudodifferential symbol of the above Dirac operator is given by

$$
\begin{aligned}
\sigma(D)(x, \xi)= & -\frac{i \gamma^{2} \sqrt{w_{1}}\left(\csc (\eta) \cos (\psi)\left(\xi_{4} \cos (\eta)-\xi_{3}\right)+\xi_{2} \sin (\psi)\right)}{\sqrt{w_{2}} \sqrt{w_{3}}} \\
& +\frac{i \gamma^{3} \sqrt{w_{2}}\left(\sin (\psi)\left(\xi_{3} \csc (\eta)-\xi_{4} \cot (\eta)\right)+\xi_{2} \cos (\psi)\right)}{\sqrt{w_{1}} \sqrt{w_{3}}} \\
& +\frac{i \gamma^{1} \xi_{1}}{\sqrt{w_{1}} \sqrt{w_{2}} \sqrt{w_{3}}}+\frac{i \gamma^{4} \xi_{4} \sqrt{w_{3}}}{\sqrt{w_{1}} \sqrt{w_{2}}}+\frac{1}{4 \sqrt{w_{1} w_{2} w_{3}}}\left(\frac{w_{1}^{\prime}}{w_{1}}+\frac{w_{2}^{\prime}}{w_{2}}+\frac{w_{3}^{\prime}}{w_{3}}\right) \gamma^{1} \\
& -\frac{\sqrt{w_{1} w_{2} w_{3}}}{4}\left(\frac{1}{w_{1}^{2}}+\frac{1}{w_{2}^{2}}+\frac{1}{w_{3}^{2}}\right) \gamma^{2} \gamma^{3} \gamma^{4} .
\end{aligned}
$$

By computing $D^{2}$ explicitly using (2.4) or by using the composition rule for symbols,

$$
\sigma\left(D^{2}\right)(x, \xi)=\sum_{\alpha \in \mathbb{Z}_{\geq 0}^{4}} \frac{(-i)^{|\alpha|}}{\alpha !} \partial_{\xi}^{\alpha} \sigma(D)(x, \xi) \partial_{x}^{\alpha} \sigma(D)(x, \xi),
$$


which is explained more generally by (3.3) and used crucially in the following section, one can find the pseudodifferential symbol of $D^{2}$ explicitly. Note that the above summation is over finitely many non-zero terms since $\sigma(D)(x, \xi)$ is a polynomial in $\xi$ whose coefficients are matrix-valued functions of the coordinates $x=\left(x^{\mu}\right) \in U$. Indeed, one finds that

$$
\sigma\left(D^{2}\right)(x, \xi)=p_{2}(x, \xi)+p_{1}(x, \xi)+p_{0}(x, \xi),
$$

where each $p_{k}$ is homogeneous of order $k$ in $\xi$. The specific expressions for the $p_{k}(x, \xi)$ are given in the appendix A.

\section{Calculation of the terms up to $a_{4}$ in the spectral action}

Calculation of the Seeley-de Witt coefficients associated with an elliptic positive differential operator on an $m$-dimensional compact manifold $M$ can be achieved by using the Cauchy integral formula and parametric pseudodifferential calculus, which is explained in detail in chapter 1 of the book [30]. Let us review this method for the operator $D^{2}$, where $D$ is the Dirac operator acting on a spin bundle on $M$. In order to derive a small time asymptotic expansion of the form

$$
\operatorname{Trace}\left(e^{-t D^{2}}\right) \sim t^{-m / 2} \sum_{n=0}^{\infty} a_{2 n}\left(D^{2}\right) t^{n} \quad\left(t \rightarrow 0^{+}\right),
$$

one can start with the Cauchy integral formula by writing

$$
e^{-t D^{2}}=\frac{1}{2 \pi i} \int_{\gamma} e^{-t \lambda}\left(D^{2}-\lambda\right)^{-1} d \lambda
$$

where the contour $\gamma$ in the complex plane goes around the non-negative real numbers clockwise. Then, the idea is to approximate $\left(D^{2}-\lambda\right)^{-1}$ by pseudodifferential operators and to derive the expansion (3.1) by computing the trace of the corresponding approximation of the heat kernel.

The symbol of $D^{2}$ in a local chart $U$ is of the form $p_{2}(x, \xi)+p_{1}(x, \xi)+p_{0}(x, \xi)$ : $U \times \mathbb{R}^{m} \rightarrow M_{k}(\mathbb{C})$, where each $p_{k}$ is homogeneous of order $k$ in $\xi$. Since $D^{2}$ is an elliptic differential operator of order 2 , the inverse of $D^{2}-\lambda$ is approximated by its parametrix $R_{\lambda}$ with

$$
\sigma\left(R_{\lambda}\right) \sim \sum_{j=0}^{\infty} r_{j}(x, \xi, \lambda),
$$

where each $r_{j}(x, \xi, \lambda)$ is a parametric pseudodifferential symbol of order $-2-j$, in the sense that

$$
r_{j}\left(x, t \xi, t^{2} \lambda\right)=t^{-2-j} r_{j}(x, \xi, \lambda) .
$$

For precise details about the type of pseudodifferential symbols used in this article, we refer the reader to page 11 and page 50 of the book [30].

We mainly use the following property of the pseudodifferential calculus. Consider pseudodifferential operators $P_{j}, j=1,2$, of orders $d_{j}$ defined in a local chart by symbols 
$\sigma\left(P_{j}\right): U \times \mathbb{R}^{m} \rightarrow M_{r}(\mathbb{C})$, namely that, for any local section $s$,

$$
\begin{aligned}
P_{j} s(x) & =(2 \pi)^{-m / 2} \int e^{i x \cdot \xi} \sigma\left(P_{j}\right)(x, \xi) \hat{s}(\xi) d \xi \\
& =(2 \pi)^{-m} \iint e^{i(x-y) \cdot \xi} \sigma\left(P_{j}\right)(x, \xi) s(y) d y d \xi .
\end{aligned}
$$

Then, the composition $P_{1} P_{2}$ is a pseudodifferential operator of order $d_{1}+d_{2}$ and its symbol is given asymptotically by (see Lemma 1.2.3 on page 17 of the book [30])

$$
\sigma\left(P_{1} P_{2}\right) \sim \sum_{\alpha \in \mathbb{Z}_{\geq 0}^{m}} \frac{(-i)^{|\alpha|}}{\alpha !} \partial_{\xi}^{\alpha} \sigma\left(P_{1}\right)(x, \xi) \partial_{x}^{\alpha} \sigma\left(P_{2}\right)(x, \xi) .
$$

It is customary and convenient to use the notation $\sigma\left(P_{1}\right) \circ \sigma\left(P_{2}\right)$ for the latter, which indeed defines a product on pseudodifferential symbols corresponding to the composition of pseudodifferential operators modulo infinitely smoothing operators.

Thus, by taking advantage of the calculus of symbols, the approximation of $\left(D^{2}-\lambda\right)^{-1}$ by the parametrix $R_{\lambda}$ turns to solving the symbolic equation

$$
\sigma\left(R_{\lambda}\left(D^{2}-\lambda\right)\right) \sim I
$$

where $I$ is the identity matrix. There is a recursive solution for the terms $r_{j}(x, \xi, \lambda)$ as follows. Considering the nuance that the parameter $\lambda$ is of order 2 (see page 51 of [30]), and using the notation $\tilde{p}_{2}=p_{2}-\lambda, \tilde{p}_{1}=p_{1}, \tilde{p}_{0}=p_{0}$, the above equation yields

$$
\begin{aligned}
\left(\sum_{j=0}^{\infty} r_{j}(x, \xi, \lambda)\right) \circ\left(\sum_{k=0}^{2} \tilde{p}_{k}\right) & \sim \sum_{k=0}^{2} \sum_{j=0}^{\infty} r_{j} \circ \tilde{p}_{k} \\
& \sim \sum_{k=0}^{2} \sum_{j=0}^{\infty} \sum_{\alpha \in \mathbb{Z}_{\geq 0}^{m}} \frac{(-i)^{|\alpha|}}{\alpha !} \partial_{\xi}^{\alpha} r_{j} \partial_{x}^{\alpha} \tilde{p}_{k} \\
& \sim \sum_{n=0}^{\infty}\left(\sum_{-2-j-|\alpha|+k=-n} \frac{(-i)^{|\alpha|}}{\alpha !} \partial_{\xi}^{\alpha} r_{j} \partial_{x}^{\alpha} \tilde{p}_{k}\right) \sim I .
\end{aligned}
$$

Note that $\partial_{\xi}^{\alpha} r_{j} \partial_{x}^{\alpha} \tilde{p}_{k}$ is of order $-2-j-|\alpha|+k$, which is a non-negative integer, and in the last summation the terms of the same homogeneity order $-n$, are gathered together.

For the case $n=0$, there is only one solution to $-2-j-|\alpha|+k=0$, which is $k=2$, $j=0, \alpha=0 \in \mathbb{Z}^{m}$, and the corresponding term in the above summation is $r_{0} \tilde{p}_{2}$. Setting this equal to $I$, which is the only homogeneous term of order 0 on the right side, one has

$$
r_{0}(x, \xi, \lambda)=\tilde{p}_{2}(x, \xi, \lambda)^{-1}=\left(p_{2}(x, \xi)-\lambda\right)^{-1} .
$$

The following argument shows that for any integer $n>0, r_{n}$ can be computed if $r_{0}, r_{1}$, $\ldots, r_{n-1}$ are given, thus, having $r_{0}$ one can proceed recursively to compute the next terms. That is, for an arbitrary arbitrary integer $n>0$, one can write the terms in the summation 
in (3.4) that are homogeneous of order $-n$ as

$$
\sum_{\substack{j \geq 0,0 \leq k \leq 2, \alpha \in \mathbb{Z}_{\geq 0}^{m} \\-2-j-|\alpha|+k=-n}} \frac{(-i)^{|\alpha|}}{\alpha !} \partial_{\xi}^{\alpha} r_{j} \partial_{x}^{\alpha} \tilde{p}_{k}=r_{n} \tilde{p}_{2}+\sum_{\substack{0 \leq j<n, 0 \leq k \leq 2, \alpha \in \mathbb{Z}_{\geq 0}^{m} \\-2-j-|\alpha|+k=-n}} \frac{(-i)^{|\alpha|}}{\alpha !} \partial_{\xi}^{\alpha} r_{j} \partial_{x}^{\alpha} \tilde{p}_{k}
$$

because in order to have $-2-j-|\alpha|+k=-n$, the conditions on the indices force that $0 \leq j \leq n$, and when $j=n$ the only solution to the equation is the case $k=2, \alpha=0 \in \mathbb{Z}^{m}$. Since the equation (3.4) indicates that the sum of the homogeneous terms of negative order should vanish, one concludes that

$$
r_{n}=-\left(\sum \frac{(-i)^{|\alpha|}}{\alpha !} \partial_{\xi}^{\alpha} r_{j} \partial_{x}^{\alpha} \tilde{p}_{k}\right)\left(\tilde{p}_{2}\right)^{-1}=-\left(\sum \frac{(-i)^{|\alpha|}}{\alpha !} \partial_{\xi}^{\alpha} r_{j} \partial_{x}^{\alpha} p_{k}\right) r_{0},
$$

where the summations are over all $\alpha \in \mathbb{Z}_{\geq 0}^{4}, j \in\{0,1, \ldots, n-1\}, k \in\{0,1,2\}$, such that $|\alpha|+j+2-k=n$. Note that in the final expression for $r_{n}$, we have replaced $\tilde{p}_{k}$ by $p_{k}$, which is allowed since in the summation, $\alpha$ has to be a non-zero tuple of non-negative integers whenever $k=2$.

By choosing a large enough $N$, the pseudodifferential operator associated with the symbol $r_{0}(x, \xi, \lambda)+\cdots+r_{N}(x, \xi, \lambda)$ provides a desired approximation of the operator $\left(D^{2}-\lambda\right)^{-1}$. By substituting this approximation in the Cauchy integral formula (3.2), one obtains an approximation of the kernel of the operator $e^{-t D^{2}}$. Then, in order to derive the asymptotic expansion for Trace $\left(e^{-t D^{2}}\right)$, one can calculate the integral of the approximation of the kernel of $e^{-t D^{2}}$ over the diagonal of $M \times M$ (against the volume form). This process requires an intricate analysis, which is carried out rigorously in section 1.7 of the book [30]. It is shown that a general coefficient in the asymptotic expansion (3.1) is given by

$$
a_{2 n}\left(D^{2}\right)=\int_{M} a_{2 n}\left(x, D^{2}\right) d \operatorname{vol}_{g}(x),
$$

where the invariantly defined function in the integral is defined in terms of the symbol $r_{2 n}$, which as shown above can be computed recursively in a local chart. That is,

$$
a_{2 n}\left(x, D^{2}\right)=\frac{(2 \pi)^{-m}}{2 \pi i} \int_{\mathbb{R}^{m}} \int_{\gamma} e^{-\lambda} \operatorname{tr}\left(r_{2 n}(x, \xi, \lambda)\right) d \lambda d^{m} \xi
$$

This shows that these coefficients are local invariants of the geometry. We note that the odd coefficients vanish since for any odd $j$, the term $r_{j}(x, \xi, \lambda)$ is an odd function of the variable $\xi \in \mathbb{R}^{m}$, whose integral over $\mathbb{R}^{m}$ vanishes.

Applying this method to $D^{2}$, where $D$ is the Dirac operator (2.4) of the Bianchi typeIX metric (2.3), we compute the corresponding $a_{0}, a_{2}, a_{4}$, which are recorded below without writing the integral with respect to the time coordinate $t$. That is, using the expressions for the homogeneous components $p_{k}$ of order $k=0,1,2$ of the symbol of $D^{2}$, which are given in the appendix A, we use the formulas (3.5) and (3.6) to explicitly compute the corresponding $r_{0}, \ldots, r_{4}$. We then use the formula (3.8) to compute the functions whose integrals over the manifold, according to the formula (3.7), give the terms $a_{0}\left(D^{2}\right), a_{2}\left(D^{2}\right)$, 
$a_{4}\left(D^{2}\right)$. It should be stressed that because of the following particular structure (cf. page 57 of the book [30])

$$
\operatorname{tr}\left(r_{n}(x, \xi, \lambda)\right)=\sum_{\substack{n=2 j-|\alpha|-2 \\|\alpha| \leq 3 n}} r_{n, j, \alpha}(x) \xi^{\alpha} \operatorname{tr}\left(r_{0}(x, \xi, \lambda)^{j}\right),
$$

which can be proved by induction from (3.5) and (3.6), the integrals involved in (3.8) can be calculated explicitly in concrete examples by using standard methods of analysis and complex analysis (indeed, the Cauchy integral formula is mainly used).

The first term, which up to multiplication by a universal constant gives the volume, is simply found to be

$$
a_{0}\left(D^{2}\right)=4 w_{1} w_{2} w_{3} .
$$

After remarkable cancelations, the next term, which up to multiplication by a universal constant gives the total curvature, is given by

$$
\begin{aligned}
a_{2}\left(D^{2}\right)= & -\frac{w_{1}^{2}}{3}-\frac{w_{2}^{2}}{3}-\frac{w_{3}^{2}}{3}+\frac{w_{1}^{2} w_{2}^{2}}{6 w_{3}^{2}}+\frac{w_{1}^{2} w_{3}^{2}}{6 w_{2}^{2}}+\frac{w_{2}^{2} w_{3}^{2}}{6 w_{1}^{2}}-\frac{\left(w_{1}^{\prime}\right)^{2}}{6 w_{1}^{2}}-\frac{\left(w_{2}^{\prime}\right)^{2}}{6 w_{2}^{2}} \\
& -\frac{\left(w_{3}^{\prime}\right)^{2}}{6 w_{3}^{2}}-\frac{w_{1}^{\prime} w_{2}^{\prime}}{3 w_{1} w_{2}}-\frac{w_{1}^{\prime} w_{3}^{\prime}}{3 w_{1} w_{3}}-\frac{w_{2}^{\prime} w_{3}^{\prime}}{3 w_{2} w_{3}}+\frac{w_{1}^{\prime \prime}}{3 w_{1}}+\frac{w_{2}^{\prime \prime}}{3 w_{2}}+\frac{w_{3}^{\prime \prime}}{3 w_{3}} .
\end{aligned}
$$

Although it seems lengthy, after an enormous amount of cancellations, the next coefficient is expressed as:

$$
\begin{aligned}
a_{4}\left(D^{2}\right) & =\frac{w_{2} w_{1}^{3}+w_{2}^{3} w_{1}}{15 w_{3}^{3}}-\frac{w_{1}^{3} w_{2}^{3}}{15 w_{3}^{5}}-\frac{w_{2} w_{3}}{15 w_{1}}+\frac{2\left(w_{1}^{\prime}\right)^{2}}{15 w_{1} w_{2} w_{3}}-\frac{w_{3}\left(w_{1}^{2}\left(w_{1}^{\prime}\right)^{2}+w_{2}^{2}\left(w_{2}^{\prime}\right)^{2}\right)}{15 w_{1}^{3} w_{2}^{3}} \\
& -\frac{7 w_{2}^{\prime} w_{3}^{\prime}}{60 w_{1}^{3}}-\frac{\left(w_{2} w_{1}^{\prime}+w_{1} w_{2}^{\prime}\right) w_{3}^{\prime}}{45 w_{1}^{2} w_{2}^{2}}+\frac{7 w_{3} w_{2}^{\prime} w_{1}^{\prime}}{90 w_{1}^{2} w_{2}^{2}}-\frac{w_{2} w_{3}\left(w_{1}^{\prime}\right)^{2}}{18 w_{1}^{5}}+\frac{5\left(w_{3} w_{2}^{\prime}+w_{2} w_{3}^{\prime}\right) w_{1}^{\prime}}{36 w_{1}^{4}} \\
& -\frac{w_{1}^{\prime \prime}}{12 w_{2} w_{3}}+\frac{w_{3} w_{2}^{\prime \prime}+w_{2} w_{3}^{\prime \prime}}{24 w_{1}^{3}}+\frac{w_{3}\left(w_{2} w_{1}^{\prime \prime}+w_{1} w_{2}^{\prime \prime}\right)}{36 w_{1}^{2} w_{2}^{2}}-\frac{5 w_{2} w_{3} w_{1}^{\prime \prime}}{72 w_{1}^{4}}-\frac{31\left(w_{1}^{\prime}\right)^{4}}{90 w_{1}^{5} w_{2} w_{3}}- \\
& \frac{41\left(w_{3} w_{2}^{\prime}+w_{2} w_{3}^{\prime}\right)\left(w_{1}^{\prime}\right)^{3}}{180 w_{1}^{4} w_{2}^{2} w_{3}^{2}}-\frac{91 w_{2}^{\prime} w_{3}^{\prime}\left(w_{1}^{\prime}\right)^{2}}{180 w_{1}^{3} w_{2}^{2} w_{3}^{2}}+\frac{5 w_{1}^{\prime \prime}\left(w_{1}^{\prime}\right)^{2}}{8 w_{1}^{4} w_{2} w_{3}}+\frac{71 w_{2}^{\prime}\left(w_{2} w_{1}^{\prime \prime}+w_{1} w_{2}^{\prime \prime}\right) w_{1}^{\prime}}{180 w_{1}^{3} w_{2}^{3} w_{3}} \\
& -\frac{w_{1}^{(3)}(t) w_{1}^{\prime}}{6 w_{1}^{3} w_{2} w_{3}}-\frac{23\left(w_{2}^{\prime}\right)^{2}\left(w_{3}^{\prime}\right)^{2}}{90 w_{1} w_{2}^{3} w_{3}^{3}}-\frac{\left(w_{1}^{\prime \prime}\right)^{2}}{6 w_{1}^{3} w_{2} w_{3}}-\frac{w_{3}\left(w_{2}^{2}\left(w_{1}^{\prime}\right)^{2}+w_{1}^{2}\left(w_{2}^{\prime}\right)^{2}\right)}{18 w_{1}^{3} w_{2}^{3}}+\frac{11 w_{2}^{\prime} w_{3}^{\prime} w_{1}^{\prime \prime}}{36 w_{1}^{2} w_{2}^{2} w_{3}^{2}} \\
& +\frac{41\left(w_{2} w_{2}^{\prime \prime}\left(w_{1}^{\prime}\right)^{2}+w_{1}\left(w_{2}^{\prime}\right)^{2} w_{1}^{\prime \prime}\right)}{360 w_{1}^{3} w_{2}^{3} w_{3}}-\frac{w_{2}^{\prime \prime} w_{3}^{\prime \prime}}{15 w_{1} w_{2}^{2} w_{3}^{2}}-\frac{w_{3}^{\prime} w_{2}^{(3)}(t)+w_{2}^{\prime} w_{3}^{(3)}(t)}{10 w_{1} w_{2}^{2} w_{3}^{2}}+\frac{w_{1}^{(4)}(t)}{30 w_{1}^{2} w_{2} w_{3}} \\
& + \text { cyclic permutations. }
\end{aligned}
$$

By cyclic permutations in the latter, we mean applying such permutations to the indices of the given expression. For the sake of clarity, the full expression of the term $a_{4}\left(D^{2}\right)$ is provided in the appendix B.

Following the conjecture of Chamseddine and Connes for Robertson-Walker metrics [11], which was addressed in [26], the crucial observation to make at this stage is that all of the coefficients appearing in the above terms are rational numbers. This indicates that the rationality result holds for the Bianchi type-IX metric, which is proved in section 5 . 


\section{Heat coefficients and the Wodzicki residue}

In this section we introduce a method for computing the Seeley-de Witt coefficients by making use of Wodzicki's noncommutative residue [54, 55]. The advantage of this method is that it involves significantly less complexity in computations, thus, for instance, it illuminates the structure of the heat expansion of the Bianchi type-IX metric more elegantly.

Given a closed $m$-dimensional manifold $M$, the Wodzicki residue is the unique trace functional on the algebra of classical pseudodifferential operators acting on the smooth sections of a vector bundle over $M$ (up to multiplication by a constant). The local symbol $\sigma$ of a classical pseudodifferential operator $P_{\sigma}$ of order $d \in \mathbb{Z}$ has an asymptotic expansion of the form

$$
\sigma(x, \xi) \sim \sum_{j=0}^{\infty} \sigma_{d-j}(x, \xi) \quad(\xi \rightarrow \infty)
$$

where each $\sigma_{d-j}: U \times\left(\mathbb{R}^{m} \backslash\{0\}\right) \rightarrow M_{r}(\mathbb{C})$ is positively homogeneous of order $d-j$ in $\xi$ in a local chart $U$ on $M$, and the endomorphisms of the vector bundle are locally identified with $M_{r}(\mathbb{C})$. The noncommutative residue of the operator $P_{\sigma}$ is defined by

$$
\operatorname{Res}\left(P_{\sigma}\right)=\int_{S^{*} M} \operatorname{tr}\left(\sigma_{-m}(x, \xi)\right) d^{m-1} \xi d^{m} x
$$

where $S^{*} M=\left\{(x, \xi) \in T^{*} M ;\|\xi\|_{g}=1\right\}$ is the cosphere bundle of $M$ and the integral is in fact the integral of the corresponding Wodzicki residue density over $M$. To be more precise, consider the volume form on the unit sphere $|\xi|=1$ in each cotangent fibre $\mathbb{R}^{m} \cong T_{x}^{*} M$,

$$
\sigma_{\xi}=\sum_{j=1}^{m}(-1)^{j-1} \xi_{j} d \xi_{1} \wedge \cdots \wedge \widehat{d} \xi_{j} \wedge \cdots \wedge d \xi_{m}
$$

The Wodzicki residue density associated with a classical pseudodifferential operator with a symbol of the form (4.1) is a 1-density on $M$ defined by

$$
\operatorname{wres}_{x} P_{\sigma}=\left(\int_{|\xi|=1} \operatorname{tr}\left(\sigma_{-m}(x, \xi)\right)\left|\sigma_{\xi}\right|\right)\left|d x^{0} \wedge d x^{1} \wedge \cdots \wedge d x^{m-1}\right| .
$$

One obtains $\operatorname{Res}\left(P_{\sigma}\right)$ by integrating this density over the manifold $M$. See $[34,54,55]$ and section 7.3 of the book [31] for more details about this residue, its equivalent spectral formulation which will be explained shortly and used crucially in this section, and the fact that, up to multiplication by a constant, it gives the unique trace functional on the algebra of classical pseudodifferential operators on a vector bundle on $M$. A crucial point is that $\operatorname{tr}\left(\sigma_{-m}(x, \xi)\right) \sigma_{\xi}$ is a closed form (see for example Proposition 7.3 on page 265 of [31]), and one can use Stokes' theorem to perform the local integrations at each point $x$ over the unit sphere $|\xi|=1$ or alternatively over the fibre of the cosphere bundle $\|\xi\|_{g}=1$.

An alternative definition for Res, which is quite spectral, provides a link between the Seeley-de Witt coefficients and the noncommutative residue. That is, for any pseudodifferential operator $P_{\sigma}$, the map that sends a complex number $s$ with a large enough real part to $\operatorname{Trace}\left(P_{\sigma} \Delta^{-s}\right)$, where $\Delta$ is a Laplacian, has a meromorphic extension to the complex 
plane with at most simple poles at its singularities. The noncommutative residue can be defined as the linear functional

$$
P_{\sigma} \mapsto \operatorname{res}_{s=0} \operatorname{Trace}\left(P_{\sigma} \Delta^{-s}\right),
$$

which turns out to be a trace functional. Thus, considering the uniqueness of the Wodzicki residue that we mentioned earlier in this section, there is a constant $c_{m}$ such that for any classical $P_{\sigma}$, we have

$$
\operatorname{Res}\left(P_{\sigma}\right)=\int_{S^{*} M} \sigma_{-m}(x, \xi) d^{m-1} \xi d^{m} x=c_{m}\left(\operatorname{res}_{s=0} \operatorname{Trace}\left(P_{\sigma} \Delta^{-s}\right)\right) .
$$

The constant $c_{m}$ can be computed easily as follows. The operator $\operatorname{Res}\left(\Delta^{-m / 2}\right)$ is of order $-m$ and its principal symbol is given by

$$
\sigma_{P}\left(\Delta^{-m / 2}\right)=\sigma_{P}\left(\Delta^{-1}\right)^{m / 2}=\sigma_{P}(\Delta)^{-m / 2}
$$

which yields

$$
\operatorname{Res}\left(\Delta^{-m / 2}\right)=\int_{S^{*} M} \operatorname{tr}\left(\sigma_{P}(\Delta)^{-m / 2}\right) d^{m-1} \xi d^{m} x
$$

On the other hand, writing $\sigma_{P}(\Delta)=\left(\sum_{i, j} g^{i j} \xi_{i} \xi_{j}\right) I$, we have

$$
\begin{aligned}
\operatorname{res}_{s=0} \operatorname{Trace}\left(\Delta^{-m / 2} \Delta^{-s}\right) & =\operatorname{res}_{s=m / 2} \operatorname{Trace}\left(\Delta^{-s}\right) \\
& =\frac{1}{\Gamma(m / 2)} \frac{(2 \pi)^{-m}}{2 \pi i} \iiint_{\gamma} e^{-\lambda} \operatorname{tr}\left(\left(\sigma_{P}(\Delta)-\lambda\right)^{-1}\right) d \lambda d^{m} \xi d^{m} x \\
& =\frac{(2 \pi)^{-m}}{\Gamma(m / 2)} \operatorname{rk}(V) \iint e^{-\sum_{i, j} g^{i j} \xi_{i} \xi_{j}} d^{m} \xi d^{m} x \\
& =\frac{(2 \pi)^{-m}}{\Gamma(m / 2)} \operatorname{rk}(V) \int \sqrt{\frac{\pi^{m}}{\operatorname{det}\left(g^{i j}\right)}} d^{m} x \\
& =\frac{2^{-m} \pi^{-m / 2}}{\Gamma(m / 2)} \operatorname{rk}(V) \int \operatorname{det}^{-1 / 2}\left(g^{i j}\right) d^{m} x,
\end{aligned}
$$

where for the second identity, we have used the formula (4.2) proved below, for $n=0$. Therefore, we have

$$
c_{m}=2^{m} \pi^{m / 2} \Gamma(m / 2) \frac{\int\left(\sum_{i, j} g^{i j} \xi_{i} \xi_{j}\right)^{-m / 2} d^{m-1} \xi d^{m} x}{\int \operatorname{det}^{-1 / 2}\left(g^{i j}\right) d^{m} x}=2^{m+1} \pi^{m} .
$$

We also need to recall from [30] the relation between small time asymptotic expansions and residues and values of corresponding zeta functions in the following sense. Using the Gamma function $\Gamma(s)=\int_{0}^{\infty} e^{-t} t^{s-1} d t$, and the Mellin transform,

$$
\lambda^{-s}=\frac{1}{\Gamma(s)} \int_{0}^{\infty} e^{-t \lambda} t^{s} \frac{d t}{t}, \quad \lambda>0,
$$


one has

$$
\operatorname{Trace}\left(\Delta^{-s}\right)=\sum \lambda_{j}^{-s}=\frac{1}{\Gamma(s)} \int_{0}^{\infty}\left(\operatorname{Trace}\left(e^{-t \Delta}\right)-\operatorname{dim} \operatorname{ker}(\Delta)\right) t^{s} \frac{d t}{t}, \quad \Re(s) \gg 0,
$$

where the summation is over all non-zero eigenvalues $\lambda_{j}$ of $\Delta$. By breaking the interval of the integration in the latter to $[0,1]$ and $(1, \infty)$, and by substituting the small time asymptotic expansion,

$$
\operatorname{Trace}\left(e^{-t \Delta}\right)=t^{-m / 2} \sum_{n=0}^{N} a_{2 n} t^{n}+O\left(t^{-m / 2+N+1}\right), \quad N \in \mathbb{Z}_{\geq 0},
$$

in the first part, one finds that

$$
\Gamma(s) \operatorname{Trace}\left(\Delta^{-s}\right)=\int_{0}^{1}\left(t^{-m / 2} \sum_{n=0}^{N} a_{2 n} t^{n}+O\left(t^{-m / 2+N+1}\right)-\operatorname{dim} \operatorname{ker}(\Delta)\right) t^{s-1} d t+h(s),
$$

where the second term,

$$
h(s)=\int_{1}^{\infty}\left(t^{-m / 2} \sum_{n=0}^{N} a_{2 n} t^{n}+O\left(t^{-m / 2+N+1}\right)-\operatorname{dim} \operatorname{ker}(\Delta)\right) t^{s-1} d t
$$

is an entire function of $s$.

Therefore, provided that $\Re(s)>m / 2$, one can write

$$
\begin{aligned}
\Gamma(s) \operatorname{Trace}\left(\Delta^{-s}\right)= & \left(\sum_{n=0}^{N} a_{2 n} \int_{0}^{1} t^{-m / 2+n+s-1} d t\right)+\int_{0}^{1} O\left(t^{-m / 2+N+1}\right) t^{s-1} d t \\
& -\operatorname{dim} \operatorname{ker}(\Delta) \int_{0}^{1} t^{s-1} d t+h(s) \\
= & \left(\sum_{n=0}^{N} \frac{a_{2 n}}{s-(m / 2-n)}\right)+\tilde{h}(s)-\frac{\operatorname{dim} \operatorname{ker}(\Delta)}{s}+h(s),
\end{aligned}
$$

where

$$
\tilde{h}(s)=\int_{0}^{1} O\left(t^{-m / 2+N+1}\right) t^{s-1} d t
$$

is a holomorphic function in the region $\Re(s)>m / 2-N-1$. By letting $N \rightarrow \infty$, this shows that the map $s \mapsto \operatorname{Trace}\left(\Delta^{-s}\right)$ defined initially for complex numbers $s$ with large enough real parts, has a meromorphic extension to the complex plane with at most simple poles located at certain points on the real line. Moreover, since $\Gamma(s)$ is holomorphic in the region $\Re(s)>0$, it follows that

$$
\operatorname{res}_{s=m / 2-n} \operatorname{Trace}\left(\Delta^{-s}\right)=\frac{a_{2 n}(\Delta)}{\Gamma(m / 2-n)},
$$

for any non-negative integer $n \leq m / 2-1$. In particular we have

$$
\operatorname{res}_{s=1} \operatorname{Trace}\left(\Delta^{-s}\right)=a_{m-2}(\Delta) .
$$

This observation yields the following assertion, which is used crucially in the sequel. 
Lemma 4.1. If $\Delta$ is a Laplacian acting on the smooth sections of a vector bundle over an $m$-dimensional manifold, then

$$
a_{m-2}(\Delta)=\frac{1}{c_{m}} \operatorname{Res}\left(\Delta^{-1}\right)=\frac{1}{2^{m+1} \pi^{m}} \operatorname{Res}\left(\Delta^{-1}\right) .
$$

Proof. It follows from the identity (4.3) and the fact that

$$
\operatorname{res}_{s=1} \operatorname{Trace}\left(\Delta^{-s}\right)=\operatorname{res}_{s=0} \operatorname{Trace}\left(\Delta^{-1} \Delta^{-s}\right)=\frac{1}{c_{m}} \operatorname{Res}\left(\Delta^{-1}\right) .
$$

Since we are mainly concerned with studying the spectral action for the Bianchi typeIX metric in this article, let us assume that $D$ is the Dirac operator on a 4-dimensional manifold. By applying Lemma (4.1) to $\Delta=D^{2}$, we have

$$
a_{2}\left(D^{2}\right)=\frac{1}{c_{4}} \operatorname{Res}\left(D^{-2}\right)=\frac{1}{32 \pi^{4}} \int_{S^{*} M} \operatorname{tr}\left(\sigma_{-4}\left(D^{-2}\right)\right) d^{3} \xi d^{4} x,
$$

where $\sigma_{-4}\left(D^{-2}\right)$ is the homogeneous component of order -4 in the expansion of the symbol of the parametrix of $D^{2}$. In the following theorem, we show that the next coefficients $a_{2 n}\left(D^{2}\right), n \geq 2$, can similarly be expressed as noncommutative residues of Laplacians.

Theorem 4.1. Let D be the Dirac operator on a 4-dimensional manifold. For any nonnegative even integer $r$, we have

$$
a_{2+r}\left(D^{2}\right)=\frac{1}{2^{5} \pi^{4+r / 2}} \operatorname{Res}\left(\Delta^{-1}\right)
$$

where

$$
\Delta=D^{2} \otimes 1+1 \otimes \Delta_{\mathbb{T}^{r}}
$$

in which $\Delta_{\mathbb{T}^{r}}$ is the flat Laplacian on the r-dimensional torus $\mathbb{T}^{r}=(\mathbb{R} / \mathbb{Z})^{r}$.

Proof. It follows from Lemma 4.1 that

$$
a_{2+r}(\Delta)=\frac{1}{c_{4+r}} \operatorname{Res}\left(\Delta^{-1}\right)
$$

Since the metric on $\mathbb{T}^{r}$ is chosen to be flat, its volume term is evidently the only non-zero heat coefficient, which combined with the Künneth formula (see part (b) of Lemma 1.7.5 on page 55 of the book [30]) implies that

$$
a_{2+r}\left(\left(x, x^{\prime}\right), \Delta\right)=a_{2+r}\left(x, D^{2}\right) a_{0}\left(x^{\prime}, \Delta_{\mathbb{T}^{r}}\right)=2^{-r} \pi^{-r / 2} a_{2+r}\left(x, D^{2}\right) .
$$

Note that, since $\sigma\left(\Delta_{\mathbb{T}^{r}}\right)\left(x^{\prime},\left(\xi_{5}, \ldots, \xi_{4+r}\right)\right)=\xi_{5}^{2}+\cdots+\xi_{4+r}^{2}$, using (3.5) and (3.8) we have

$$
a_{0}\left(x^{\prime}, \Delta_{\mathbb{T}^{r}}\right)=2^{-r} \pi^{-r / 2} .
$$

Therefore, by integrating (4.4), we obtain

$$
a_{2+r}\left(D^{2}\right)=2^{r} \pi^{r / 2} a_{2+r}(\Delta)=\frac{2^{r} \pi^{r / 2}}{c_{4+r}} \operatorname{Res}\left(\Delta^{-1}\right)=\frac{1}{2^{5} \pi^{4+r / 2}} \operatorname{Res}\left(\Delta^{-1}\right) .
$$


A direct consequence of this theorem provides an efficient method for computing the Seeley-de Witt coefficients with significantly less complexities in the calculations. It also yields an elegant proof of the rationality result for the Bianchi type-IX metric, which is presented in the following section.

Corollary 4.1. Assuming the conditions and notations of Theorem 4.1 and using the symbol $\xi^{\prime} \in \mathbb{R}^{4+r}$ for a covector in the cotangent fibre at $\left(x, x^{\prime}\right) \in U \times \mathbb{T}^{r}$ in a local chart, we have

$$
a_{2+r}\left(D^{2}\right)=\frac{1}{2^{5} \pi^{4+r / 2}} \int_{S^{*}\left(M \times \mathbb{T}^{r}\right)} \operatorname{tr}\left(\sigma_{-4-r}\left(\Delta^{-1}\right)\right) d^{3+r} \xi^{\prime} d^{4} x,
$$

where in the local chart, $\sigma_{-4-r}\left(\Delta^{-1}\right):\left(U \times \mathbb{T}^{r}\right) \times \mathbb{R}^{4+r} \rightarrow M_{4}(\mathbb{C})$ is the homogeneous component of order $-4-r$ in the asymptotic expansion of the symbol of the parametrix $\Delta^{-1}$ of $\Delta=D^{2} \otimes 1+1 \otimes \Delta_{\mathbb{T}^{r}}$.

Proof. It follows from the fact that if

$$
\sigma\left(D^{2}\right)(x, \xi)=p_{2}(x, \xi)+p_{1}(x, \xi)+p_{0}(x, \xi): U \times \mathbb{R}^{4} \rightarrow M_{4}(\mathbb{C}),
$$

where each $p_{k}$ is homogeneous of order $k$ in $\xi$, then $\sigma(\Delta):\left(U \times \mathbb{T}^{r}\right) \times \mathbb{R}^{4+r} \rightarrow M_{4}(\mathbb{C})$ is given by

$$
\sigma(\Delta)\left(\left(x, x^{\prime}\right),\left(\xi_{1}, \ldots, \xi_{4}, \xi_{5}, \ldots, \xi_{4+r}\right)\right)=p_{2}^{\prime}+p_{1}^{\prime}+p_{0}^{\prime}
$$

where

$p_{2}^{\prime}=p_{2}(x, \xi)+\left(\xi_{5}^{2}+\cdots+\xi_{4+r}^{2}\right) I, \quad p_{1}^{\prime}=p_{1}(x, \xi), \quad p_{0}^{\prime}=p_{0}(x, \xi), \quad(x, \xi) \in U \times \mathbb{R}^{4}$

Clearly each $p_{k}^{\prime}=p_{k}^{\prime}\left(\left(x, x^{\prime}\right), \xi^{\prime}\right)$ is defined on $\left(U \times \mathbb{T}^{r}\right) \times \mathbb{R}^{4+r}$ with values in $M_{4}(\mathbb{C})$, and it is homogeneous of order $k$ in $\xi^{\prime}$. Using Theorem 4.1,

$$
\begin{aligned}
a_{2+r}\left(D^{2}\right) & =\frac{1}{2^{5} \pi^{4+r / 2}} \operatorname{Res}\left(\Delta^{-1}\right) \\
& =\frac{1}{2^{5} \pi^{4+r / 2}} \int_{S^{*}\left(M \times \mathbb{T}^{r}\right)} \operatorname{tr}\left(\sigma_{-4-r}\left(\Delta^{-1}\right)\left(\left(x, x^{\prime}\right), \xi^{\prime}\right)\right) d^{3+r} \xi^{\prime} d^{4} x d^{r} x^{\prime},
\end{aligned}
$$

where $\sigma_{-4-r}\left(\Delta^{-1}\right)$ is the homogeneous component of order $-4-r$ in $\xi^{\prime}=\left(\xi, \xi_{5}, \ldots, \xi_{4+r}\right) \in$ $\mathbb{R}^{4+r}$ in the asymptotic expansion of the symbol of the parametrix $\Delta^{-1}$ of $\Delta$. We claim that $\sigma_{-4-r}\left(\Delta^{-1}\right)$ is independent of the coordinate $x^{\prime} \in \mathbb{T}^{r}$, thus, combined with the fact $\operatorname{Vol}\left(\mathbb{T}^{r}\right)=1$, it follows from the equation (4.5) that

$$
a_{2+r}\left(D^{2}\right)=\frac{1}{2^{5} \pi^{4+r / 2}} \int_{S^{*}\left(M \times \mathbb{T}^{r}\right)} \operatorname{tr}\left(\sigma_{-4-r}\left(\Delta^{-1}\right)\left(\left(x, x^{\prime}\right), \xi^{\prime}\right)\right) d^{3+r} \xi^{\prime} d^{4} x .
$$

The claimed fact about the independence of $\sigma_{-4-r}\left(\Delta^{-1}\right)\left(\left(x, x^{\prime}\right), \xi^{\prime}\right)$ from $x^{\prime}$ can be justified easily from the independence of the symbols $p_{k}^{\prime}$ from $x^{\prime}$. However, since it will be used crucially in the following section, we find explicit recursive formulas for all homogeneous terms $\sigma_{-2-j}\left(\Delta^{-1}\right)$ of order $-2-j$ in $\xi^{\prime}$ in the expansion $\sigma\left(\Delta^{-1}\right) \sim \sum_{j=0}^{\infty} \sigma_{-2-j}\left(\Delta^{-1}\right)$, 
which will in particular prove their independence from $x^{\prime}$. Using the composition rule (3.3) we have

$$
\begin{aligned}
\sigma\left(\Delta^{-1}\right) \circ \sigma(\Delta) & =\left(\sum_{j=0}^{\infty} \sigma_{-2-j}\left(\Delta^{-1}\right)\right) \circ \sum_{k=0}^{2} p_{k}^{\prime} \\
& \sim \sum_{j=0}^{\infty} \sum_{k=0}^{2} \sum_{\alpha \in \mathbb{Z}_{\geq 0}^{4}} \sum_{\beta \in \mathbb{Z}_{\geq 0}^{r}} \frac{(-i)^{|\alpha|+|\beta|}}{\alpha ! \beta !}\left(\partial_{\xi^{\prime}}^{(\alpha, \beta)} \sigma_{-2-j}\left(\Delta^{-1}\right)\right)\left(\partial_{x^{\prime}}^{\beta} \partial_{x}^{\alpha} p_{k}^{\prime}\right) \\
& \sim \sum_{n=0}^{\infty}\left(\sum_{-2-j-|\alpha|-|\beta|+k=-n} \frac{(-i)^{|\alpha|+|\beta|}}{\alpha ! \beta !}\left(\partial_{\xi^{\prime}}^{(\alpha, \beta)} \sigma_{-2-j}\left(\Delta^{-1}\right)\right)\left(\partial_{x^{\prime}}^{\beta} \partial_{x}^{\alpha} p_{k}^{\prime}\right)\right) \sim I .
\end{aligned}
$$

Note that $\left(\partial_{\xi^{\prime}}^{(\alpha, \beta)} \sigma_{-2-j}\left(\Delta^{-1}\right)\right)\left(\partial_{x^{\prime}}^{\beta} \partial_{x}^{\alpha} p_{k}^{\prime}\right)$ is a symbol of order $-2-j-|\alpha|-|\beta|+k$, which is a non-positive integer, and in the last summation the symbols of the same order $-n$ are gathered together. The zero order term corresponds to the case $n=0$, for which the equation $-2-j-|\alpha|-|\beta|+k=0$ has only one solution, namely $k=2, j=0, \alpha=0 \in \mathbb{Z}_{\geq 0}^{4}$, $\beta=0 \in \mathbb{Z}_{\geq 0}^{r}$. Therefore, by comparing the homogeneous terms on the two sides of the equation, we have $\sigma_{-2}\left(\Delta^{-1}\right) p_{2}^{\prime}=I$, which gives

$$
\sigma_{-2}\left(\Delta^{-1}\right)\left(\left(x, x^{\prime}\right), \xi^{\prime}\right)=\left(p_{2}^{\prime}\right)^{-1}=\left(p_{2}(x, \xi)+\left(\xi_{5}^{2}+\cdots+\xi_{4+r}^{2}\right) I\right)^{-1}
$$

and the following argument shows that each $\sigma_{-2-n}\left(\Delta^{-1}\right)$ is recursively computable once $\sigma_{-2}\left(\Delta^{-1}\right), \sigma_{-3}\left(\Delta^{-1}\right), \ldots, \sigma_{-2-n+1}\left(\Delta^{-1}\right)$ are computed. The reason is that when $j=n$, the only solution to $-2-j-|\alpha|-|\beta|+k=-n$ is $k=2, \alpha=0 \in \mathbb{Z}_{\geq 0}^{4}, \beta=0 \in \mathbb{Z}_{\geq 0}^{r}$. Therefore the terms that are of order $-n$ in the above equation can be written as

$$
\sigma_{-2-n}\left(\Delta^{-1}\right) p_{2}^{\prime}+\sum_{\substack{0 \leq j<n, 0 \leq k \leq 2 \\ \alpha \in \mathbb{Z}_{20}^{4}, \beta \in \mathbb{Z}_{\geq 0}^{r} \\-2-j-|\alpha|-|\beta|+\bar{k}=-n}} \frac{(-i)^{|\alpha|+|\beta|}}{\alpha ! \beta !}\left(\partial_{\xi^{\prime}}^{(\alpha, \beta)} \sigma_{-2-j}\left(\Delta^{-1}\right)\right)\left(\partial_{x^{\prime}}^{\beta} \partial_{x}^{\alpha} p_{k}^{\prime}\right)
$$

Setting this expression equal to 0 for $n>0$, and using $\left(p_{2}^{\prime}\right)^{-1}=\sigma_{-2}\left(\Delta^{-1}\right)$ we have

$$
\left.\begin{array}{rl}
\sigma_{-2-n}\left(\Delta^{-1}\right)\left(\left(x, x^{\prime}\right), \xi^{\prime}\right) \\
=-\left(\sum_{\substack{0 \leq j<n, 0 \leq k \leq 2 \\
\alpha \in \mathbb{Z}_{\geq 0}^{4}, \beta \in \mathbb{Z}_{\geq 0}^{r} \\
-2-j-|\alpha|-|\beta|+k=-n}} \frac{(-i)^{|\alpha|+|\beta|}}{\alpha ! \beta !}\left(\partial_{\xi^{\prime}}^{(\alpha, \beta)} \sigma_{-2-j}\left(\Delta^{-1}\right)\right)\left(\partial_{x^{\prime}}^{\beta} \partial_{x}^{\alpha} p_{k}^{\prime}\right)\right) \sigma_{-2}\left(\Delta^{-1}\right) .
\end{array}\right)
$$

Note that in the latter, since $p_{k}^{\prime}$ has no dependence on $x^{\prime}$, for any $\beta \neq 0 \in \mathbb{Z}_{\geq 0}^{r}$ the corresponding term in the summation is 0 . Moreover, when $k=2$, since $j<n, \alpha$ is a non-zero 4-tuple of non-negative integers, which means that $\partial_{x}^{\alpha} p_{k}^{\prime}=\partial_{x}^{\alpha} p_{k}$. Therefore for 
any $n>0$ we have

$$
\sigma_{-2-n}\left(\Delta^{-1}\right)\left(\left(x, x^{\prime}\right), \xi^{\prime}\right)=-\left(\sum_{\substack{0 \leq j<n, 0 \leq k \leq 2 \\ \alpha \in \mathbb{Z}_{\geq 0}^{\leq} \leq 0 \\-2-j-|\alpha|+k=-n}} \frac{(-i)^{|\alpha|}}{\alpha !}\left(\partial_{\xi}^{\alpha} \sigma_{-2-j}\left(\Delta^{-1}\right)\right)\left(\partial_{x}^{\alpha} p_{k}\right)\right) \sigma_{-2}\left(\Delta^{-1}\right),
$$

which is seen by induction to have no dependence on $x^{\prime}$.

We confirm the validity of the coefficients $a_{0}, a_{2}, a_{4}$, calculated for the Bianchi type-IX metric in section 3 by noting that the method devised in the present section produces the same expressions. We stress that in practice the new method is significantly more convenient since the expression that leads to a Seeley-de Witt coefficient simplifies when one considers its restriction to the corresponding cosphere bundle in order to compute the noncommutative residue.

The noncommutative residue was originally discovered in the 1-dimensional case by Adler [1] and Manin [39]. Its coincidence with the Dixmier trace [13] on pseudodifferential operators of order $-m$ on an $m$-dimensional closed manifold indicates its applicability for explicit and convenient computations. It is also worth mentioning that a noncommutative residue developed for noncommutative tori [27, 28] simplified a purely noncommutative heat kernel computation significantly and clarified in [25] the reason for mysterious and remarkable cancellations that occur in this type of computations.

\section{Rationality of the spectral action for Bianchi type-IX metrics}

In this section we prove that the Seeley-de Witt coefficients $a_{2 n}\left(D^{2}\right)$ appearing in the expansion of the spectral action for the Bianchi type-IX metric are expressed in terms of several variable polynomials with rational coefficients evaluated on the cosmic evolution factors $w_{1}(t), w_{2}(t), w_{3}(t)$, and their derivatives of certain orders. This extends the statement conjectured in [11] and addressed in [26] for Robertson-Walker metrics to a homogeneous anisotropic cosmological model.

In order to prove the rationality result for the Bianchi type-IX metric, similar to the treatment in [26], let us start with the crucial observation that the local forms $a_{2 n}\left(x, D^{2}\right) d^{3} x$, where $D$ is the Dirac operator of this geometry, are invariant over the spatial manifold $\mathbb{S}^{3}$. This can be seen from the defining formula (1.1) for the metric, in which the left invariance of the 1 -forms $\sigma_{1}, \sigma_{2}, \sigma_{3}$, implies that the metric is invariant under any diffeomorphism arising from left multiplication by an element of $\mathrm{SU}(2)$. Since the action is transitive and left multiplication by an element of $\mathrm{SU}(2)$ is an isometry, any isometry-invariant function on $\mathbb{S}^{3}$ is independent of the spatial coordinates. In particular, the restriction of the kernel of $e^{-t D^{2}}$ to the diagonal and consequently the differential forms $a_{2 n}\left(x, D^{2}\right) d^{4} x$ are invariant, and if we set $a_{2 n}\left(x, D^{2}\right) d^{3} x=\tilde{a}_{2 n}\left(x, D^{2}\right) d v o l_{g}$, where $d v o l_{g}$ is the volume form, then $\tilde{a}_{2 n}\left(x, D^{2}\right)$ is independent of the spatial coordinates for any $n$. 
Furthermore, we can easily determine the general form of $\tilde{a}_{2 n}\left(x, D^{2}\right)$ by applying the method devised in section 4 , which is based on making use of the noncommutative residue and the Künneth formula and restricting the computations to the cosphere bundle. This method is summarized in Corollary 4.1. In order to use this statement for our purpose, which is finding out the general form of $\tilde{a}_{2 n}\left(x, D^{2}\right)$ for the Bianchi type-IX metric, we choose $r$ such that $2+r=2 n$. Then, we need to consider the homogeneous term $\sigma_{-2-2 n}\left(\left(x, x^{\prime}\right), \xi^{\prime}\right)$ of order $-2 n-2=-4-r$ in the expansion of the symbol of the operator $\Delta=D^{2} \otimes 1+1 \otimes$ $\Delta_{\mathbb{T}^{2 n-2}}$. Note that, as it is shown in the proof of Corollary $4.1, \sigma_{-2-2 n}$ is independent of the coordinate $x^{\prime} \in \mathbb{T}^{2 n-2}$, and it is homogeneous in $\xi^{\prime}=\left(\xi, \xi_{5}, \ldots, \xi_{4+r}\right) \in \mathbb{R}^{4+r} \backslash\{0\}$ (in accordance with the notation used in the proof, we consider $\left.\xi \in \mathbb{R}^{4}\right)$. Thus, in the sequel we simply write $\sigma_{-2-2 n}\left(x, \xi^{\prime}\right)$ instead of $\sigma_{-2-2 n}\left(\left(x, x^{\prime}\right), \xi^{\prime}\right)$.

Now if we set $\zeta_{\mu+1}=\sum_{\nu} e_{\mu}^{\nu} \xi_{\nu+1}^{\prime}$, then considering the explicit recursive formula (4.7), which starts from (4.6), and by using the explicit expressions given in the appendix B for the components of the symbol of $D^{2}$, it can be seen by induction that for any integer $n \geq 1$,

$$
\left.\sigma_{-2-2 n}\left(x, \xi^{\prime}\right)\right|_{S^{*}\left(M \times \mathbb{T}^{2 n-2}\right)}=\left.\sigma_{-2-2 n}\left(x, \xi^{\prime}(\zeta)\right)\right|_{\zeta \in \mathbb{S}^{2 n+1}}=\left(w_{1} w_{2} w_{3}\right)^{-3 n} P_{2 n}(\zeta),
$$

where $P_{2 n}(\zeta)$ is a polynomial in $\zeta_{1}, \ldots, \zeta_{2 n+2}$, with the following property. The coefficients of the polynomial $P_{2 n}$ are matrices whose entries are in the algebra generated by rational numbers, trigonometric functions of the spatial coordinates, and $w_{i}^{(p)}$ where $i \in\{1,2,3\}$, $p \in\{0,1, \ldots, 2 n\}$. This fact leads to the following statement about the general form of the coefficients $a_{2 n}\left(D^{2}\right)$.

Theorem 5.1. For any non-negative integer $n$, the coefficient $a_{2 n}\left(D^{2}\right)$ in the expansion of the spectral action for the Bianchi type-IX metric is of the form

$$
a_{2 n}\left(D^{2}\right)=\left(w_{1} w_{2} w_{3}\right)^{1-3 n} Q_{2 n}\left(w_{1}, w_{2}, w_{3}, w_{1}^{\prime}, w_{2}^{\prime}, w_{3}^{\prime}, \ldots, w_{1}^{(2 n)}, w_{2}^{(2 n)}, w_{3}^{(2 n)}\right),
$$

where $Q_{2 n}$ is a polynomial with rational coefficients.

Proof. It follows from Corollary 4.1 that

$$
\begin{aligned}
a_{2+r}\left(x, D^{2}\right) & =\frac{1}{2^{5} \pi^{4+r / 2}} \int_{S^{*}\left(M \times \mathbb{T}^{r}\right)} \operatorname{tr}\left(\sigma_{-4-r}\left(\Delta^{-1}\right)\right) d^{3+r} \xi^{\prime} \\
& =\frac{1}{2^{5} \pi^{4+r / 2}} \int_{\mathbb{S}^{3+r}} \operatorname{tr}\left(\sigma_{-4-r}\left(\Delta^{-1}\right)\right) d v o l_{g} d^{3+r} \zeta,
\end{aligned}
$$

where, as above $\zeta_{\mu+1}=\sum_{\nu} e_{\mu}^{\nu} \xi_{\nu+1}^{\prime}$ so that the Jacobian of the coordinate transformation is just $d v^{\prime} l_{g}$. This implies that

$$
\tilde{a}_{2 n}\left(x, D^{2}\right)=\frac{1}{2^{5} \pi^{n+3}} \int_{\mathbb{S}^{2 n+1}} \operatorname{tr}\left(\sigma_{-2-2 n}\left(\Delta^{-1}\right)\right) d^{2 n+1} \zeta,
$$

which, as shown above, is independent of the spatial coordinates. Thus, we have

$$
\begin{aligned}
a_{2 n}\left(D^{2}\right) & =\int \tilde{a}_{2 n}\left(x, D^{2}\right) d \operatorname{vol}_{g}=\operatorname{Vol} \cdot \tilde{a}_{2 n}\left(D^{2}\right)=16 \pi^{2} w_{1} w_{2} w_{3} \tilde{a}_{2 n}\left(D^{2}\right) \\
& =\frac{w_{1} w_{2} w_{3}}{2 \pi^{n+1}} \int_{\mathbb{S}^{2 n+1}} \operatorname{tr}\left(\sigma_{-2-2 n}\left(\Delta^{-1}\right)\right) d^{2 n+1} \zeta .
\end{aligned}
$$


The equation (5.1) allows us to write $\sigma_{-2-2 n}\left(x, \xi^{\prime}(\zeta)\right)=\left(w_{1} w_{2} w_{3}\right)^{-3 n} P_{2 n}(\zeta)$, which yields

$$
\begin{aligned}
a_{2 n}\left(D^{2}\right) & \left.=\frac{\left(w_{1} w_{2} w_{3}\right)^{1-3 n}}{2 \pi^{n+1}} \int_{\mathbb{S}^{2 n+1}} \operatorname{tr}\left(P_{2 n}(\zeta)\right)\left(\Delta^{-1}\right)\right) d^{2 n+1} \zeta \\
& =\left(w_{1} w_{2} w_{3}\right)^{1-3 n} Q_{2 n}\left(w_{1}, w_{2}, w_{3}, w_{1}^{\prime}, w_{2}^{\prime}, w_{3}^{\prime}, \ldots, w_{1}^{(2 n)}, w_{2}^{(2 n)}, w_{3}^{(2 n)}\right) .
\end{aligned}
$$

Note that $\operatorname{tr}\left(P_{2 n}(\zeta)\right)$ is a polynomial in $\zeta_{1}, \zeta_{2}, \ldots, \zeta_{2 n+2}$, with the coefficients in the algebra generated by the rational numbers, trigonometric functions of the spatial coordinates, and $w_{i}^{(p)}$ where $i \in\{1,2,3\}, p \in\{0,1, \ldots, 2 n\}$.

The integral of a monomial $m_{\alpha}(\zeta)=c_{\alpha} \zeta_{1}^{\alpha_{1}} \cdots \zeta_{2 n+2}^{\alpha_{2 n+2}}$ over $\mathbb{S}^{2 n+1}$ is either 0 , or can be written as

$$
\int_{\mathbb{S}^{2 n+1}} m_{\alpha}(\zeta) d^{2 n+1} \zeta=\frac{2 c_{\alpha} \prod_{j} \Gamma\left(\frac{\alpha_{j}+1}{2}\right)}{\Gamma\left(n+1+\frac{|\alpha|}{2}\right)},
$$

if each $\alpha_{j}$ is an even non-negative integer. Also, recall that $\Gamma\left(\frac{n}{2}\right)=q \pi^{\frac{1}{2}}$ for some $q \in \mathbb{Q}$ when $n \in 2 \mathbb{N}+1$, and $\Gamma\left(\frac{n}{2}\right) \in \mathbb{Z}$ when $n \in 2 \mathbb{N}$. Therefore we have

$$
\int_{\mathbb{S}^{2 n+1}} m_{\alpha}(\zeta) d^{2 n+1} \zeta=q \pi^{\frac{2 n+2}{2}}=q \pi^{n+1}
$$

for some $q \in \mathbb{Q}$ if $c_{\alpha} \in \mathbb{Q}$. Since $a_{2 n}\left(D^{2}\right)=\left(w_{1} w_{2} w_{3}\right)^{1-3 n} Q_{2 n}$ has no spatial dependence, we conclude that

$$
\left.Q_{2 n}=\frac{1}{2 \pi^{n+1}} \int_{\mathbb{S}^{2 n+1}} \operatorname{tr}\left(P_{2 n}(\zeta)\right)\left(\Delta^{-1}\right)\right) d^{2 n+1} \zeta
$$

belongs to the algebra generated by the $w_{i}^{(p)}$ and rational numbers.

\section{Gravitational instantons, modular forms, and rationality}

Among the Euclidean Bianchi type-IX models, an especially interesting class consists of the Bianchi IX gravitational instantons. A gravitational instanton is both self-dual (that is, the Weyl curvature tensor is self-dual) and an Einstein metric (the Ricci tensor is proportional to the metric). A remarkable feature of Bianchi IX gravitational instantons with SU(2) symmetry is that they can be completely classified in terms of solutions to Painlevé VI integrable systems, $[32,49,53]$. The latter are a 4-parameter family of singular ordinary differential equations of the form

$$
\begin{aligned}
\frac{d^{2} X}{d t^{2}}= & \frac{1}{2}\left(\frac{1}{X}+\frac{1}{X-1}+\frac{1}{X-t}\right)\left(\frac{d X}{d t}\right)^{2}-\left(\frac{1}{t}+\frac{1}{t-1}+\frac{1}{X-t}\right) \frac{d X}{d t} \\
& +\frac{X(X-1)(X-t)}{t^{2}(t-1)^{2}}\left(\alpha+\beta \frac{t}{X^{2}}+\gamma \frac{t-1}{(X-1)^{2}}+\delta \frac{t(t-1)}{(X-t)^{2}}\right) .
\end{aligned}
$$

The self-dual equation for the $\mathrm{SU}(2)$ Bianchi IX metrics is written in [49] as an ordinary differential equation in the $w_{i}$ and in additional functions $\alpha_{i}, i=1,2,3$ that arise as the components of the connection 1-form in a basis of anti-self-dual 2-forms, see [53]. In terms 
of the conformally invariant variable $x=\left(\alpha_{2}-\alpha_{1}\right)\left(\alpha_{2}-\alpha_{3}\right)^{-1}$ the self-dual equations for the Riemannian Bianchi IX metric can be rephrased as a system of equations

$$
w_{i}=\Omega_{i} x^{\prime}(x(1-x))^{-1 / 2}, \quad \Omega_{1}^{\prime}=-\frac{\Omega_{2} \Omega_{3}}{x(1-x)}, \quad \Omega_{2}^{\prime}=-\frac{\Omega_{3} \Omega_{1}}{x}, \quad \Omega_{3}^{\prime}=-\frac{\Omega_{1} \Omega_{2}}{1-x} .
$$

These in turn can then be reduced to a case of the Painlevé VI equation with parameters

$$
(\alpha, \beta, \gamma, \delta)=\left(\frac{1}{8},-\frac{1}{8}, \frac{1}{8}, \frac{3}{8}\right)
$$

see $[49,53]$. In [3], the solutions to this equation are given explicitly in terms of a parameterization involving theta functions and theta characteristics

$$
\vartheta[p, q](z, i \mu):=\sum_{m \in \mathbb{Z}} \exp \left(-\pi(m+p)^{2} \mu+2 \pi i(m+p)(z+q)\right) .
$$

Namely, with the notation $\vartheta[p, q]:=\vartheta[p, q](0, i \mu)$, and

$$
\vartheta_{2}:=\vartheta[1 / 2,0], \quad \vartheta_{3}:=\vartheta[0,0], \quad \vartheta_{4}:=\vartheta[0,1 / 2],
$$

one finds $\alpha_{i}=2 \partial_{\mu} \log \vartheta_{i+1}$ and

$$
w_{1}=-\frac{i}{2} \vartheta_{3} \vartheta_{4} \frac{\frac{\partial}{\partial q} \vartheta\left[p, q+\frac{1}{2}\right]}{e^{\pi i p} \vartheta[p, q]}, \quad w_{2}=\frac{i}{2} \vartheta_{2} \vartheta_{4} \frac{\frac{\partial}{\partial q} \vartheta\left[p+\frac{1}{2}, q+\frac{1}{2}\right]}{e^{\pi i p} \vartheta[p, q]}, \quad w_{3}=-\frac{1}{2} \vartheta_{2} \vartheta_{3} \frac{\frac{\partial}{\partial q} \vartheta\left[p+\frac{1}{2}, q\right]}{\vartheta[p, q]} .
$$

The asymptotics of these solutions were analyzed in [40], where it is shown that, for large $\mu$, they approximate Eguchi-Hanson type gravitational instantons with $w_{2}=w_{3} \neq w_{1}$, [20].

It is clear that, for the Bianchi IX gravitational instantons, using the parameterization of [3], the Seeley-de Witt coefficients $a_{2 n}$ of the spectral action are rational functions, with $\mathbb{Q}$-coefficients, in the $\vartheta_{2}, \vartheta_{3}, \vartheta_{4}, \vartheta[p, q], \partial_{q} \vartheta[p, q]$ and $e^{i \pi p}$ and derivatives, hence they belong to the field generated, over $\mathbb{Q}$, by these functions. We will return in a second part of this work [23] to discuss in detail the arithmetic properties of the spectral action for Bianchi IX gravitational instantons.

In this perspective, one can view the rationality question about the spectral action in a similar light to analogous questions that occur whenever arithmetic and number theoretic structures arise in theoretical physics. For example, when Feynman integrals are interpreted as periods (see [41] for an overview of that setting), the fact that the relevant amplitude forms and domains of integration are algebraic over $\mathbb{Q}($ or $\mathbb{Z})$ has direct implications on the class of numbers that arise as periods. Another such instance of arithmetic structures in physics, where rational coefficients play an important role, is in the zero temperature KMS states of quantum statistical mechanical systems: in the case constructed in [17] (see also chapter 3 of [18]) for instance, the construction of an arithmetic algebra of observables, defined over $\mathbb{Q}$, is linked to modular functions and makes it possible to have KMS states with values in the modular field. The relation between the spectral action of Bianchi IX gravitational instantons and modular forms will be discussed in [23]. 


\section{Conclusions}

We have shown that the Seeley-de Witt coefficients $a_{2 n}\left(D^{2}\right)$ associated with the Dirac operator $D$ of the Bianchi type-IX metric, which appear in the expansion of the spectral action [7], are expressed by polynomials with rational coefficients evaluated on the cosmic evolution factors $w_{1}(t), w_{2}(t), w_{3}(t)$, and their derivatives of certain orders. It is quite interesting that although this metric provides a homogeneous anisotropic cosmological model, after remarkable cancellations, only rational coefficients appear in the final expression for each $a_{2 n}\left(D^{2}\right)$. Such a rationality result was first conjectured in [11] for Robertson-Walker metrics, which was addressed in [26].

Our proof of the rationality statement for the Bianchi type-IX model, similar to the argument given in [26], begins with the crucial observation that the kernel of $e^{-t D^{2}}$ is restricted to have no spatial dependence on the diagonal. We then take a novel approach to proceed the argument. That is, we have devised a general method that expresses the Seeley-de Witt coefficients of a geometry as noncommutative residues of operators. This is an efficient method that allows explicit calculations with significantly less complexities, compared to the method of using parametric pseudodifferential calculus [30]. More importantly, it leads to an elegant proof of the rationality result for the Bianchi type-IX metric. To be more explicit, the Wodzicki residue [54,55] involves an integration over the cosphere bundle of a manifold, and the expression for computing $a_{2 n}\left(D^{2}\right)$ simplifies to our favor when restricted to the cosphere bundle, in the view of our method.

A main application of the explicit formulae for the spectral action of the Bianchi IX minisuperspace models obtained in this paper will be given in the forthcoming second part of this work [23]: we will use our explicit formulae, along with the parameterization of the Bianchi IX gravitational instantons in terms of solutions of Painlevé VI equations, to show that the spectral action for these Euclidean signature spacetimes is expressible in terms of vector valued modular forms, of the type that occurs in the Eichler-Zagier theory of Jacobi forms [21]. The rationality result we proved in this paper will play a crucial role, as it will reveal an underlying (and otherwise invisible) arithmetic structure, with the coefficients of the asymptotic expansion being rational combinations of modular forms.

\section{A Pseudodifferential symbol of $D^{2}$}

The Dirac operator $D$ of the Bianchi type-IX metric is computed explicitly in section 2 and its expression is given by (2.4). This allows to find the pseudodifferential symbol $\sigma(D): U \times \mathbb{R}^{4} \rightarrow M_{4}(\mathbb{C})$ of this operator in the local chart explicitly, which is given by (2.5). However, in order to employ the methods explained in section 3 and section 4 for computing the Seeley-de Witt coefficients, it is necessary to compute the symbol of $D^{2}$. This can be achieved in two different ways. First, one can use the expression (2.4) to obtain an explicit formula for $D^{2}$ and thereby obtain its pseudodifferential symbol. Note that $(2.2)$ makes it clear how, by means of the Fourier transform and the Fourier inversion formula, one can express a pseudodifferential operator and in particular a differential operator in terms of its symbol. Second, one can use the composition rule (3.3) for the composition of 
symbols, which reduces to (2.6) for the computation of

$$
\sigma\left(D^{2}\right)(x, \xi)=p_{2}(x, \xi)+p_{1}(x, \xi)+p_{0}(x, \xi),
$$

where each $p_{k}: U \times \mathbb{R}^{4} \rightarrow M_{4}(\mathbb{C})$ is homogeneous of order $k$ in $\xi$.

We find that the principal part of $\sigma\left(D^{2}\right)$ is given by

$$
\begin{aligned}
p_{2}(x, \xi)= & \frac{1}{w_{1} w_{2} w_{3}}\left(\xi_{4}^{2} w_{1}^{2} \cot ^{2}(\eta) \cos ^{2}(\psi)+\xi_{3}^{2} w_{1}^{2} \csc ^{2}(\eta) \cos ^{2}(\psi)+\xi_{2} \xi_{4} w_{1}^{2} \cot (\eta) \sin (2 \psi)\right. \\
& -\xi_{2} \xi_{3} w_{1}^{2} \csc (\eta) \sin (2 \psi)-2 \xi_{3} \xi_{4} w_{1}^{2} \cot (\eta) \csc (\eta) \cos ^{2}(\psi)+\xi_{2}^{2} w_{1}^{2} \sin ^{2}(\psi) \\
& +\xi_{4}^{2} w_{2}^{2} \cot ^{2}(\eta) \sin ^{2}(\psi)-\xi_{2} \xi_{4} w_{2}^{2} \cot (\eta) \sin (2 \psi)+\xi_{3}^{2} w_{2}^{2} \csc ^{2}(\eta) \sin ^{2}(\psi)+ \\
& \left.\xi_{2} \xi_{3} w_{2}^{2} \csc (\eta) \sin (2 \psi)-2 \xi_{3} \xi_{4} w_{2}^{2} \cot (\eta) \csc (\eta) \sin ^{2}(\psi)+\xi_{2}^{2} w_{2}^{2} \cos ^{2}(\psi)+\xi_{4}^{2} w_{3}^{2}+\xi_{1}^{2}\right) I,
\end{aligned}
$$

where $I$ is the $4 \times 4$ identity matrix. The component of $\sigma\left(D^{2}\right)$ that is homogeneous of order 1 has a lengthy expression:

$$
\begin{aligned}
& p_{1}(x, \xi)=\left(-\frac{i \xi_{2} w_{1} \cot (\eta) \cos ^{2}(\psi)}{w_{2} w_{3}}-\frac{i \xi_{2} w_{2} \cot (\eta) \sin ^{2}(\psi)}{w_{1} w_{3}}-\frac{3 i \xi_{4} w_{2} \csc ^{2}(\eta) \sin (2 \psi)}{4 w_{1} w_{3}}+\right. \\
& \frac{3 i \xi_{4} w_{1} \csc ^{2}(\eta) \sin (2 \psi)}{4 w_{2} w_{3}}-\frac{i \xi_{4} w_{2} \cos (2 \eta) \csc ^{2}(\eta) \sin (2 \psi)}{4 w_{1} w_{3}}+\frac{i \xi_{4} w_{1} \cos (2 \eta) \csc ^{2}(\eta) \sin (2 \psi)}{4 w_{2} w_{3}} \\
& \left.+\frac{i \xi_{3} w_{2} \cot (\eta) \csc (\eta) \sin (2 \psi)}{w_{1} w_{3}}-\frac{i \xi_{3} w_{1} \cot (\eta) \csc (\eta) \sin (2 \psi)}{w_{2} w_{3}}\right) I \\
& +\left(\frac{i \xi_{4} w_{3}}{2 w_{1}^{2}}+\frac{i \xi_{4} w_{3}}{2 w_{2}^{2}}-\frac{i \xi_{4}}{2 w_{3}}\right) \gamma^{2} \gamma^{3}+\left(\frac{i \xi_{4} w_{2} \cot (\eta) \sin (\psi)}{2 w_{1}^{2}}-\frac{i \xi_{3} w_{2} \csc (\eta) \sin (\psi)}{2 w_{1}^{2}}\right. \\
& -\frac{i \xi_{2} w_{2} \cos (\psi)}{2 w_{1}^{2}}+\frac{i \xi_{4} w_{2} \cot (\eta) \sin (\psi)}{2 w_{3}^{2}}-\frac{i \xi_{3} w_{2} \csc (\eta) \sin (\psi)}{2 w_{3}^{2}}-\frac{i \xi_{2} w_{2} \cos (\psi)}{2 w_{3}^{2}} \\
& \left.-\frac{i \xi_{4} \cot (\eta) \sin (\psi)}{2 w_{2}}+\frac{i \xi_{3} \csc (\eta) \sin (\psi)}{2 w_{2}}+\frac{i \xi_{2} \cos (\psi)}{2 w_{2}}\right) \gamma^{2} \gamma^{4} \\
& +\left(-\frac{i \xi_{4} w_{1} \cot (\eta) \cos (\psi)}{2 w_{2}^{2}}+\frac{i \xi_{3} w_{1} \csc (\eta) \cos (\psi)}{2 w_{2}^{2}}-\frac{i \xi_{2} w_{1} \sin (\psi)}{2 w_{2}^{2}}-\frac{i \xi_{4} w_{1} \cot (\eta) \cos (\psi)}{2 w_{3}^{2}}\right. \\
& +\frac{i \xi_{3} w_{1} \csc (\eta) \cos (\psi)}{2 w_{3}^{2}}-\frac{i \xi_{2} w_{1} \sin (\psi)}{2 w_{3}^{2}}+\frac{i \xi_{4} \cot (\eta) \cos (\psi)}{2 w_{1}}-\frac{i \xi_{3} \csc (\eta) \cos (\psi)}{2 w_{1}} \\
& \left.+\frac{i \xi_{2} \sin (\psi)}{2 w_{1}}\right) \gamma^{3} \gamma^{4}+\left(-\frac{i \xi_{4} w_{1}^{\prime}}{2 w_{1}^{2} w_{2}}-\frac{i \xi_{4} w_{2}^{\prime}}{2 w_{1} w_{2}^{2}}+\frac{i \xi_{4} w_{3}^{\prime}}{2 w_{1} w_{2} w_{3}}\right) \gamma^{1} \gamma^{4} \\
& +\left(-\frac{i \xi_{4} \cot (\eta) \cos (\psi) w_{1}^{\prime}}{2 w_{1} w_{2} w_{3}}+\frac{i \xi_{3} \csc (\eta) \cos (\psi) w_{1}^{\prime}}{2 w_{1} w_{2} w_{3}}-\frac{i \xi_{2} \sin (\psi) w_{1}^{\prime}}{2 w_{1} w_{2} w_{3}}+\frac{i \xi_{4} \cot (\eta) \cos (\psi) w_{2}^{\prime}}{2 w_{2}^{2} w_{3}}\right. \\
& -\frac{i \xi_{3} \csc (\eta) \cos (\psi) w_{2}^{\prime}}{2 w_{2}^{2} w_{3}}+\frac{i \xi_{2} \sin (\psi) w_{2}^{\prime}}{2 w_{2}^{2} w_{3}}+\frac{i \xi_{4} \cot (\eta) \cos (\psi) w_{3}^{\prime}}{2 w_{2} w_{3}^{2}}-\frac{i \xi_{3} \csc (\eta) \cos (\psi) w_{3}^{\prime}}{2 w_{2} w_{3}^{2}} \\
& \left.+\frac{i \xi_{2} \sin (\psi) w_{3}^{\prime}}{2 w_{2} w_{3}^{2}}\right) \gamma^{1} \gamma^{2} \\
& +\left(\frac{i \xi_{4} \cot (\eta) \sin (\psi) w_{1}^{\prime}}{2 w_{1}^{2} w_{3}}-\frac{i \xi_{3} \csc (\eta) \sin (\psi) w_{1}^{\prime}}{2 w_{1}^{2} w_{3}}-\frac{i \xi_{2} \cos (\psi) w_{1}^{\prime}}{2 w_{1}^{2} w_{3}}-\frac{i \xi_{4} \cot (\eta) \sin (\psi) w_{2}^{\prime}}{2 w_{1} w_{2} w_{3}}\right. \\
& +\frac{i \xi_{3} \csc (\eta) \sin (\psi) w_{2}^{\prime}}{2 w_{1} w_{2} w_{3}}+\frac{i \xi_{2} \cos (\psi) w_{2}^{\prime}}{2 w_{1} w_{2} w_{3}}+\frac{i \xi_{4} \cot (\eta) \sin (\psi) w_{3}^{\prime}}{2 w_{1} w_{3}^{2}}-\frac{i \xi_{3} \csc (\eta) \sin (\psi) w_{3}^{\prime}}{2 w_{1} w_{3}^{2}} \\
& \left.-\frac{i \xi_{2} \cos (\psi) w_{3}^{\prime}}{2 w_{1} w_{3}^{2}}\right) \gamma^{1} \gamma^{3} \text {. }
\end{aligned}
$$


Finally, we have the zero order part of $\sigma\left(D^{2}\right)$ :

$$
\begin{aligned}
p_{0}(x, \xi)= & \left(-\frac{w_{1}^{\prime}}{8 w_{1} w_{2}^{2}}-\frac{w_{1}^{\prime}}{8 w_{1} w_{3}^{2}}+\frac{3 w_{1}^{\prime}}{8 w_{1}^{3}}-\frac{w_{2}^{\prime}}{8 w_{1}^{2} w_{2}}-\frac{w_{3}^{\prime}}{8 w_{1}^{2} w_{3}}-\frac{w_{2}^{\prime}}{8 w_{2} w_{3}^{2}}+\frac{3 w_{2}^{\prime}}{8 w_{2}^{3}}-\frac{w_{3}^{\prime}}{8 w_{2}^{2} w_{3}}\right. \\
& \left.+\frac{3 w_{3}^{\prime}}{8 w_{3}^{3}}\right) \gamma^{1} \gamma^{2} \gamma^{3} \gamma^{4}+\left(-\frac{w_{1}^{\prime \prime}}{4 w_{1}^{2} w_{2} w_{3}}+\frac{w_{1}^{\prime} w_{2}^{\prime}}{8 w_{1}^{2} w_{2}^{2} w_{3}}+\frac{w_{1}^{\prime} w_{3}^{\prime}}{8 w_{1}^{2} w_{2} w_{3}^{2}}+\frac{5 w_{1}^{\prime 2}}{16 w_{1}^{3} w_{2} w_{3}}-\frac{w_{2}^{\prime \prime}}{4 w_{1} w_{2}^{2} w_{3}}\right. \\
& +\frac{w_{2}^{\prime} w_{3}^{\prime}}{8 w_{1} w_{2}^{2} w_{3}^{2}}+\frac{5 w_{2}^{\prime 2}}{16 w_{1} w_{2}^{3} w_{3}}-\frac{w_{3}^{\prime \prime}}{4 w_{1} w_{2} w_{3}^{2}}+\frac{5 w_{3}^{\prime 2}}{16 w_{1} w_{2} w_{3}^{3}}+\frac{w_{2} w_{3}}{16 w_{1}^{3}}+\frac{w_{3}}{8 w_{1} w_{2}}+\frac{w_{1} w_{3}}{16 w_{2}^{3}} \\
& \left.+\frac{w_{2}}{8 w_{1} w_{3}}+\frac{w_{1}}{8 w_{2} w_{3}}+\frac{w_{1} w_{2}}{16 w_{3}^{3}}\right) I .
\end{aligned}
$$

\section{B Full expression of the term $a_{4}\left(D^{2}\right)$}

The expression (3.9) for the term $a_{4}\left(D^{2}\right)$, where $D$ is the Dirac operator of the Bianchi type-IX metric, is given in a short form by indicating that one needs to apply cyclic permutations to the indices of the given expression and add the results to the expression. For the sake of clarity the full expression of this term is provided here:

$$
\begin{aligned}
& a_{4}\left(D^{2}\right)=-\frac{w_{1}^{3} w_{2}^{3}}{15 w_{3}^{5}}-\frac{w_{1}^{3} w_{3}^{3}}{15 w_{2}^{5}}-\frac{w_{2}^{3} w_{3}^{3}}{15 w_{1}^{5}}+\frac{w_{1}^{3} w_{2}}{15 w_{3}^{3}}+\frac{w_{1} w_{2}^{3}}{15 w_{3}^{3}}+\frac{w_{1}^{3} w_{3}}{15 w_{2}^{3}}+\frac{w_{2}^{3} w_{3}}{15 w_{1}^{3}}+\frac{w_{1} w_{3}^{3}}{15 w_{2}^{3}} \\
& +\frac{w_{2} w_{3}^{3}}{15 w_{1}^{3}}-\frac{w_{1} w_{2}}{15 w_{3}}-\frac{w_{1} w_{3}}{15 w_{2}}-\frac{w_{2} w_{3}}{15 w_{1}}-\frac{w_{2}\left(w_{1}^{\prime}\right)^{2}}{15 w_{1} w_{3}^{3}}-\frac{w_{3}\left(w_{1}^{\prime}\right)^{2}}{15 w_{1} w_{2}^{3}}-\frac{w_{3}\left(w_{2}^{\prime}\right)^{2}}{15 w_{1}^{3} w_{2}} \\
& -\frac{w_{1}\left(w_{2}^{\prime}\right)^{2}}{15 w_{2} w_{3}^{3}}-\frac{w_{1}\left(w_{3}^{\prime}\right)^{2}}{15 w_{2}^{3} w_{3}}-\frac{w_{2}\left(w_{3}^{\prime}\right)^{2}}{15 w_{1}^{3} w_{3}}+\frac{2\left(w_{1}^{\prime}\right)^{2}}{15 w_{1} w_{2} w_{3}}+\frac{2\left(w_{2}^{\prime}\right)^{2}}{15 w_{1} w_{2} w_{3}} \\
& +\frac{2\left(w_{3}^{\prime}\right)^{2}}{15 w_{1} w_{2} w_{3}}-\frac{w_{2}\left(w_{1}^{\prime}\right)^{2}}{18 w_{1}^{3} w_{3}}-\frac{w_{3}\left(w_{1}^{\prime}\right)^{2}}{18 w_{1}^{3} w_{2}}-\frac{w_{1}\left(w_{2}^{\prime}\right)^{2}}{18 w_{2}^{3} w_{3}}-\frac{w_{3}\left(w_{2}^{\prime}\right)^{2}}{18 w_{1} w_{2}^{3}} \\
& -\frac{w_{1}\left(w_{3}^{\prime}\right)^{2}}{18 w_{2} w_{3}^{3}}-\frac{w_{2}\left(w_{3}^{\prime}\right)^{2}}{18 w_{1} w_{3}^{3}}-\frac{w_{2} w_{3}\left(w_{1}^{\prime}\right)^{2}}{18 w_{1}^{5}}-\frac{w_{1} w_{3}\left(w_{2}^{\prime}\right)^{2}}{18 w_{2}^{5}}-\frac{w_{1} w_{2}\left(w_{3}^{\prime}\right)^{2}}{18 w_{3}^{5}} \\
& -\frac{31\left(w_{1}^{\prime}\right)^{4}}{90 w_{1}^{5} w_{2} w_{3}}-\frac{31\left(w_{2}^{\prime}\right)^{4}}{90 w_{1} w_{2}^{5} w_{3}}-\frac{31\left(w_{3}^{\prime}\right)^{4}}{90 w_{1} w_{2} w_{3}^{5}}-\frac{7 w_{1}^{\prime} w_{2}^{\prime}}{60 w_{3}^{3}}-\frac{7 w_{1}^{\prime} w_{3}^{\prime}}{60 w_{2}^{3}}-\frac{7 w_{2}^{\prime} w_{3}^{\prime}}{60 w_{1}^{3}} \\
& -\frac{w_{1}^{\prime} w_{2}^{\prime}}{45 w_{1}^{2} w_{3}}-\frac{w_{1}^{\prime} w_{2}^{\prime}}{45 w_{2}^{2} w_{3}}-\frac{w_{2}^{\prime} w_{3}^{\prime}}{45 w_{1} w_{3}^{2}}+\frac{5 w_{3} w_{1}^{\prime} w_{2}^{\prime}}{36 w_{1}^{4}}+\frac{5 w_{3} w_{1}^{\prime} w_{2}^{\prime}}{36 w_{2}^{4}}+\frac{5 w_{2} w_{1}^{\prime} w_{3}^{\prime}}{36 w_{1}^{4}} \\
& +\frac{5 w_{2} w_{1}^{\prime} w_{3}^{\prime}}{36 w_{3}^{4}}+\frac{5 w_{1} w_{2}^{\prime} w_{3}^{\prime}}{36 w_{2}^{4}}+\frac{5 w_{1} w_{2}^{\prime} w_{3}^{\prime}}{36 w_{3}^{4}}+\frac{7 w_{3} w_{1}^{\prime} w_{2}^{\prime}}{90 w_{1}^{2} w_{2}^{2}}+\frac{7 w_{2} w_{1}^{\prime} w_{3}^{\prime}}{90 w_{1}^{2} w_{3}^{2}}+\frac{7 w_{1} w_{2}^{\prime} w_{3}^{\prime}}{90 w_{2}^{2} w_{3}^{2}} \\
& -\frac{41\left(w_{1}^{\prime}\right)^{3} w_{2}^{\prime}}{180 w_{1}^{4} w_{2}^{2} w_{3}}-\frac{41 w_{1}^{\prime}\left(w_{2}^{\prime}\right)^{3}}{180 w_{1}^{2} w_{2}^{4} w_{3}}-\frac{41\left(w_{1}^{\prime}\right)^{3} w_{3}^{\prime}}{180 w_{1}^{4} w_{2} w_{3}^{2}}-\frac{41 w_{1}^{\prime}\left(w_{3}^{\prime}\right)^{3}}{180 w_{1}^{2} w_{2} w_{3}^{4}}-\frac{41 w_{2}^{\prime}\left(w_{3}^{\prime}\right)^{3}}{180 w_{1} w_{2}^{2} w_{3}^{4}} \\
& -\frac{41\left(w_{2}^{\prime}\right)^{3} w_{3}^{\prime}}{180 w_{1} w_{2}^{4} w_{3}^{2}}-\frac{23\left(w_{1}^{\prime}\right)^{2}\left(w_{2}^{\prime}\right)^{2}}{90 w_{1}^{3} w_{2}^{3} w_{3}}-\frac{23\left(w_{1}^{\prime}\right)^{2}\left(w_{3}^{\prime}\right)^{2}}{90 w_{1}^{3} w_{2} w_{3}^{3}}-\frac{23\left(w_{2}^{\prime}\right)^{2}\left(w_{3}^{\prime}\right)^{2}}{90 w_{1} w_{2}^{3} w_{3}^{3}} \\
& -\frac{w_{1}^{\prime} w_{3}^{\prime}}{45 w_{1}^{2} w_{2}}-\frac{w_{1}^{\prime} w_{3}^{\prime}}{45 w_{2} w_{3}^{2}}-\frac{w_{2}^{\prime} w_{3}^{\prime}}{45 w_{1} w_{2}^{2}}-\frac{91\left(w_{1}^{\prime}\right)^{2} w_{2}^{\prime} w_{3}^{\prime}}{180 w_{1}^{3} w_{2}^{2} w_{3}^{2}}-\frac{91 w_{1}^{\prime}\left(w_{2}^{\prime}\right)^{2} w_{3}^{\prime}}{180 w_{1}^{2} w_{2}^{3} w_{3}^{2}} \\
& -\frac{91 w_{1}^{\prime} w_{2}^{\prime}\left(w_{3}^{\prime}\right)^{2}}{180 w_{1}^{2} w_{2}^{2} w_{3}^{3}}+\frac{w_{2} w_{1}^{\prime \prime}}{24 w_{3}^{3}}+\frac{w_{3} w_{1}^{\prime \prime}}{24 w_{2}^{3}}+\frac{w_{1} w_{2}^{\prime \prime}}{24 w_{3}^{3}}+\frac{w_{3} w_{2}^{\prime \prime}}{24 w_{1}^{3}}+\frac{w_{1} w_{3}^{\prime \prime}}{24 w_{2}^{3}}+\frac{w_{2} w_{3}^{\prime \prime}}{24 w_{1}^{3}} \\
& -\frac{w_{1}^{\prime \prime}}{12 w_{2} w_{3}}-\frac{w_{2}^{\prime \prime}}{12 w_{1} w_{3}}-\frac{w_{3}^{\prime \prime}}{12 w_{1} w_{2}}+\frac{w_{2} w_{1}^{\prime \prime}}{36 w_{1}^{2} w_{3}}+\frac{w_{3} w_{1}^{\prime \prime}}{36 w_{1}^{2} w_{2}}+\frac{w_{1} w_{2}^{\prime \prime}}{36 w_{2}^{2} w_{3}}
\end{aligned}
$$




$$
\begin{aligned}
& -\frac{5 w_{2} w_{3} w_{1}^{\prime \prime}}{72 w_{1}^{4}}-\frac{5 w_{1} w_{3} w_{2}^{\prime \prime}}{72 w_{2}^{4}}-\frac{5 w_{1} w_{2} w_{3}^{\prime \prime}}{72 w_{3}^{4}}+\frac{5\left(w_{1}^{\prime}\right)^{2} w_{1}^{\prime \prime}}{8 w_{1}^{4} w_{2} w_{3}}+\frac{5\left(w_{2}^{\prime}\right)^{2} w_{2}^{\prime \prime}}{8 w_{1} w_{2}^{4} w_{3}} \\
& +\frac{5\left(w_{3}^{\prime}\right)^{2} w_{3}^{\prime \prime}}{8 w_{1} w_{2} w_{3}^{4}}+\frac{71 w_{1}^{\prime} w_{2}^{\prime} w_{1}^{\prime \prime}}{180 w_{1}^{3} w_{2}^{2} w_{3}}+\frac{71 w_{1}^{\prime} w_{2}^{\prime} w_{2}^{\prime \prime}}{180 w_{1}^{2} w_{2}^{3} w_{3}}+\frac{71 w_{1}^{\prime} w_{3}^{\prime} w_{1}^{\prime \prime}}{180 w_{1}^{3} w_{2} w_{3}^{2}}+\frac{71 w_{1}^{\prime} w_{3}^{\prime} w_{3}^{\prime \prime}}{180 w_{1}^{2} w_{2} w_{3}^{3}} \\
& +\frac{71 w_{2}^{\prime} w_{3}^{\prime} w_{3}^{\prime \prime}}{180 w_{1} w_{2}^{2} w_{3}^{3}}+\frac{71 w_{2}^{\prime} w_{3}^{\prime} w_{2}^{\prime \prime}}{180 w_{1} w_{2}^{3} w_{3}^{2}}+\frac{41\left(w_{2}^{\prime}\right)^{2} w_{1}^{\prime \prime}}{360 w_{1}^{2} w_{2}^{3} w_{3}}+\frac{41\left(w_{3}^{\prime}\right)^{2} w_{1}^{\prime \prime}}{360 w_{1}^{2} w_{2} w_{3}^{3}}+\frac{41\left(w_{2}^{\prime}\right)^{2} w_{3}^{\prime \prime}}{360 w_{1} w_{2}^{3} w_{3}^{2}} \\
& +\frac{41\left(w_{3}^{\prime}\right)^{2} w_{2}^{\prime \prime}}{360 w_{1} w_{2}^{2} w_{3}^{3}}+\frac{41\left(w_{1}^{\prime}\right)^{2} w_{2}^{\prime \prime}}{360 w_{1}^{3} w_{2}^{2} w_{3}}+\frac{41\left(w_{1}^{\prime}\right)^{2} w_{3}^{\prime \prime}}{360 w_{1}^{3} w_{2} w_{3}^{2}}+\frac{11 w_{2}^{\prime} w_{3}^{\prime} w_{1}^{\prime \prime}}{36 w_{1}^{2} w_{2}^{2} w_{3}^{2}}+\frac{11 w_{1}^{\prime} w_{3}^{\prime} w_{2}^{\prime \prime}}{36 w_{1}^{2} w_{2}^{2} w_{3}^{2}} \\
& +\frac{11 w_{1}^{\prime} w_{2}^{\prime} w_{3}^{\prime \prime}}{36 w_{1}^{2} w_{2}^{2} w_{3}^{2}}-\frac{\left(w_{1}^{\prime \prime}\right)^{2}}{6 w_{1}^{3} w_{2} w_{3}}-\frac{\left(w_{2}^{\prime \prime}\right)^{2}}{6 w_{1} w_{2}^{3} w_{3}}-\frac{\left(w_{3}^{\prime \prime}\right)^{2}}{6 w_{1} w_{2} w_{3}^{3}}+\frac{w_{3} w_{2}^{\prime \prime}}{36 w_{1} w_{2}^{2}} \\
& +\frac{w_{1} w_{3}^{\prime \prime}}{36 w_{2} w_{3}^{2}}+\frac{w_{2} w_{3}^{\prime \prime}}{36 w_{1} w_{3}^{2}}-\frac{w_{1}^{\prime \prime} w_{2}^{\prime \prime}}{15 w_{1}^{2} w_{2}^{2} w_{3}}-\frac{w_{2}^{\prime \prime} w_{3}^{\prime \prime}}{15 w_{1} w_{2}^{2} w_{3}^{2}}-\frac{w_{1}^{\prime \prime} w_{3}^{\prime \prime}}{15 w_{1}^{2} w_{2} w_{3}^{2}}-\frac{w_{1}^{\prime} w_{1}(3)}{6 w_{1}^{3} w_{2} w_{3}} \\
& -\frac{w_{2}^{\prime} w_{2}{ }^{(3)}}{6 w_{1} w_{2}^{3} w_{3}}-\frac{w_{3}^{\prime} w_{3}{ }^{(3)}}{6 w_{1} w_{2} w_{3}^{3}}-\frac{w_{2}^{\prime} w_{1}{ }^{(3)}}{10 w_{1}^{2} w_{2}^{2} w_{3}}-\frac{w_{3}^{\prime} w_{1}{ }^{(3)}}{10 w_{1}^{2} w_{2} w_{3}^{2}}-\frac{w_{1}^{\prime} w_{2}{ }^{(3)}}{10 w_{1}^{2} w_{2}^{2} w_{3}}-\frac{w_{3}^{\prime} w_{2}{ }^{(3)}}{10 w_{1} w_{2}^{2} w_{3}^{2}} \\
& -\frac{w_{1}^{\prime} w_{3}^{(3)}}{10 w_{1}^{2} w_{2} w_{3}^{2}}-\frac{w_{2}^{\prime} w_{3}{ }^{(3)}}{10 w_{1} w_{2}^{2} w_{3}^{2}}+\frac{w_{1}^{(4)}}{30 w_{1}^{2} w_{2} w_{3}}+\frac{w_{2}^{(4)}}{30 w_{1} w_{2}^{2} w_{3}}+\frac{w_{3}{ }^{(4)}}{30 w_{1} w_{2} w_{3}^{2}} .
\end{aligned}
$$

\section{Acknowledgments}

The first author is supported by a Summer Undergraduate Research Fellowship at Caltech. The third author is partially supported by NSF grants DMS-1201512 and PHY-1205440 and by the Perimeter Institute for Theoretical Physics.

Open Access. This article is distributed under the terms of the Creative Commons Attribution License (CC-BY 4.0), which permits any use, distribution and reproduction in any medium, provided the original author(s) and source are credited.

\section{References}

[1] M. Adler, On a trace functional for formal pseudo differential operators and the symplectic structure of the Korteweg-de Vries type equations, Invent. Math. 50 (1978) 219.

[2] L. Álvarez-Gaumé, Supersymmetry and the Atiyah-Singer Index Theorem, Commun. Math. Phys. 90 (1983) 161 [INSPIRE].

[3] M.V. Babich and D.A. Korotkin, Selfdual SU(2) invariant Einstein metrics and modular dependence of theta functions, Lett. Math. Phys. 46 (1998) 323 [gr-qc/9810025] [INSPIRE].

[4] A. Ball and M. Marcolli, Spectral Action Models of Gravity on Packed Swiss Cheese Cosmology, arXiv: 1506.01401 [INSPIRE].

[5] B. Ćácić, M. Marcolli and K. Teh, Coupling of gravity to matter, spectral action and cosmic topology, J. Noncommut. Geom. 8 (2014) 473. [arXiv:1106.5473] [INSPIRE].

[6] A.H. Chamseddine and A. Connes, Universal formula for noncommutative geometry actions: Unification of gravity and the standard model, Phys. Rev. Lett. 77 (1996) 4868 [INSPIRE].

[7] A.H. Chamseddine and A. Connes, The spectral action principle, Commun. Math. Phys. 186 (1997) 731 [hep-th/9606001] [INSPIRE]. 
[8] A.H. Chamseddine and A. Connes, Conceptual Explanation for the Algebra in the Noncommutative Approach to the Standard Model, Phys. Rev. Lett. 99 (2007) 191601 [arXiv:0706.3690] [INSPIRE].

[9] A.H. Chamseddine and A. Connes, Quantum Gravity Boundary Terms from Spectral Action, Phys. Rev. Lett. 99 (2007) 071302 [arXiv:0705.1786] [INSPIRE].

[10] A.H. Chamseddine and A. Connes, The Uncanny Precision of the Spectral Action, Commun. Math. Phys. 293 (2010) 867 [arXiv:0812.0165] [InSPIRE].

[11] A.H. Chamseddine and A. Connes, Spectral Action for Robertson-Walker metrics, JHEP 10 (2012) 101 [arXiv:1105.4637] [INSPIRE].

[12] A.H. Chamseddine, A. Connes and M. Marcolli, Gravity and the standard model with neutrino mixing, Adv. Theor. Math. Phys. 11 (2007) 991 [hep-th/0610241] [INSPIRE].

[13] A. Connes, The Action Functional in Noncommutative Geometry, Commun. Math. Phys. 117 (1988) 673 [INSPIRE].

[14] A. Connes, Noncommutative geometry, Academic Press, (1994).

[15] A. Connes, Noncommutative geometry and the standard model with neutrino mixing, JHEP 11 (2006) 081 [hep-th/0608226] [INSPIRE].

[16] A. Connes, On the spectral characterization of manifolds, J. Noncommut. Geom. 7 (2013) 1 [arXiv:0810.2088] [INSPIRE].

[17] A. Connes and M. Marcolli, Quantum Statistical Mechanics of $\mathbb{Q}$-lattices, in Frontiers in number theory, physics, and geometry. I, Springer, Berlin, (2006), pg. 269-347.

[18] A. Connes and M. Marcolli, Noncommutative Geometry, Quantum Fields and Motives, American Mathematical Society Colloquium Publications, vol. 55, (2008).

[19] P.D. D'Eath, Supersymmetric Quantum Cosmology, Cambridge University Press, (2005).

[20] T. Eguchi and A.J. Hanson, Selfdual Solutions to Euclidean Gravity, Annals Phys. 120 (1979) 82 [inSPIRE].

[21] M. Eichler and D. Zagier, The theory of Jacobi forms, Progress in Mathematics, vol. 55, Birkhäuser, (1985).

[22] C. Estrada and M. Marcolli, Noncommutative Mixmaster Cosmologies, Int. J. Geom. Meth. Mod. Phys. 10 (2013) 1250086 [arXiv:1203.2679] [InSPIRE].

[23] W. Fan, F. Fathizadeh and M. Marcolli, in preparation.

[24] L. Fang and R. Ruffini eds., Quantum Cosmology, World Scientific, (1987).

[25] F. Fathizadeh, On the Scalar Curvature for the Noncommutative Four Torus, J. Math. Phys. 56 (2015) 062303 [arXiv:1410.8705].

[26] F. Fathizadeh, A. Ghorbanpour and M. Khalkhali, Rationality of Spectral Action for Robertson-Walker Metrics, JHEP 12 (2014) 064 [arXiv:1407.5972] [INSPIRE].

[27] F. Fathizadeh and M. Khalkhali, Scalar Curvature for Noncommutative Four-Tori, J. Noncommut. Geom. 9 (2015) 473 [arXiv:1301.6135].

[28] F. Fathizadeh and M.W. Wong, Noncommutative residues for pseudo-differential operators on the noncommutative two-torus, J. Pseudo-Differ. Oper. Appl. 2 (2011) 289. 
[29] T. Friedrich, Dirac operators in Riemannian geometry, American Mathematical Society, (2000).

[30] P. Gilkey, Invariance theory, the heat equation, and the Atiyah-Singer index theorem, Mathematics Lecture Series, vol. 11, Publish or Perish Inc., Wilmington, DE, (1984).

[31] J.M. Gracia-Bondia, J.C. Várilly and H. Figueroa, Elements of noncommutative geometry, Birkhäuser, (2001).

[32] N.J. Hitchin, Twistor spaces, Einstein metrics and isomonodromic deformations, J. Diff. Geom. 42 (1995) 30.

[33] W. Kalau and M. Walze, Gravity, noncommutative geometry and the Wodzicki residue, J. Geom. Phys. 16 (1995) 327 [gr-qc/9312031] [INSPIRE].

[34] C. Kassel, Le résidu non commutatif (d'après M. Wodzicki), Séminaire Bourbaki, vol. 1988/89, Astérisque No. 177-178 (1989), Exp. No. 708, 199-229.

[35] D. Kastler, The Dirac operator and gravitation, Commun. Math. Phys. 166 (1995) 633 [INSPIRE].

[36] D. Kolodrubetz and M. Marcolli, Boundary conditions of the RGE flow in the noncommutative geometry approach to particle physics and cosmology, Phys. Lett. B 693 (2010) 166 [arXiv:1006.4000] [InSPIRE].

[37] K.V. Kuchar and M.P. Ryan, Is minisuperspace quantization valid?: Taub in mixmaster, Phys. Rev. D 40 (1989) 3982 [inSPIRE].

[38] H.B. Lawson and M.-L. Michelsohn, Spin geometry, Princeton University Press, (1989).

[39] Ju.I. Manin, Algebraic aspects of nonlinear differential equations (in Russian), Current problems in mathematics, vol. 11 (in Russian), pg. 5-152, (errata insert) Akad. Nauk SSSR Vsesojuz. Inst. Naucn. i Tehn. Informacii, Moscow, (1978).

[40] Y. Manin and M. Marcolli, Symbolic Dynamics, Modular Curves and Bianchi IX Cosmologies, arXiv:1504.04005 [INSPIRE].

[41] M. Marcolli, Feynman motives, World Scientific Publishing Co. Pte. Ltd., (2010).

[42] M. Marcolli, Building cosmological models via noncommutative geometry, Int. J. Geom. Methods Mod. Phys. 8 (2011) 1131 [inSPIRE].

[43] M. Marcolli and E. Pierpaoli, Early Universe models from Noncommutative Geometry, Adv. Theor. Math. Phys. 14 (2010) [arXiv:0908.3683] [INSPIRE].

[44] M. Marcolli, E. Pierpaoli and K. Teh, The coupling of topology and inflation in Noncommutative Cosmology, Commun. Math. Phys. 309 (2012) 341 [arXiv: 1012.0780] [INSPIRE].

[45] M. Marcolli, E. Pierpaoli and K. Teh, The spectral action and cosmic topology, Commun. Math. Phys. 304 (2011) 125 [arXiv:1005.2256] [INSPIRE].

[46] W. Nelson, J. Ochoa and M. Sakellariadou, Constraining the Noncommutative Spectral Action via Astrophysical Observations, Phys. Rev. Lett. 105 (2010) 101602 [arXiv: 1005.4279] [INSPIRE].

[47] W. Nelson and M. Sakellariadou, Natural inflation mechanism in asymptotic noncommutative geometry, Phys. Lett. B 680 (2009) 263 [arXiv:0903.1520] [INSPIRE]. 
[48] W. Nelson and M. Sakellariadou, Cosmology and the Noncommutative approach to the Standard Model, Phys. Rev. D 81 (2010) 085038 [arXiv:0812.1657] [INSPIRE].

[49] S. Okumura, The self-dual Einstein-Weyl metric and classical solution of Painlevé VI, Lett. Math. Phys. 46 (1998) 219.

[50] J. Roe, Elliptic operators, topology and asymptotic methods, second edition, Longman, (1998).

[51] S. Sinha and B.L. Hu, Validity of the minisuperspace approximation: An example from interacting quantum field theory, Phys. Rev. D 44 (1991) 1028 [InSPIRE].

[52] K. Teh, Nonperturbative spectral action of round coset spaces of $\mathrm{SU}(2)$, J. Noncommut. Geom. 7 (2013) 677 [arXiv:1010.1827].

[53] K.P. Tod, Self-dual Einstein metrics from the Painlevé VI equation, Phys. Lett. A 190 (1994) 221.

[54] M. Wodzicki, Local invariants of spectral asymmetry, Invent. Math. 75 (1984) 143.

[55] M. Wodzicki, Noncommutative residue. I. Fundamentals, Springer, Berlin, K-theory, arithmetic and geometry, (Moscow, 1984-1986), Lect. Notes Math. 1289 (1987) 320.

[56] E. Witten, Supersymmetry and Morse Theory, J. Diff. Geom. 17 (1982) 661. 